\title{
A Systematic Review and Meta-analysis of Neuroimaging in Oppositional Defiant Disorder (ODD) and Conduct Disorder (CD) Taking Attention-Deficit Hyperactivity Disorder (ADHD) Into Account
}

\author{
Siri D. S. Noordermeer ${ }^{1}$ (D) Marjolein Luman $^{1} \cdot$ Jaap Oosterlaan $^{1}$
}

Received: 28 August 2015 / Accepted: 18 December 2015 / Published online: 5 February 2016

(C) The Author(s) 2016. This article is published with open access at Springerlink.com

\begin{abstract}
Oppositional defiant disorder (ODD) and conduct disorder (CD) are common behavioural disorders in childhood and adolescence and are associated with brain abnormalities. This systematic review and meta-analysis investigates structural (sMRI) and functional MRI (fMRI) findings in individuals with ODD/CD with and without attention-deficit hyperactivity disorder (ADHD). Online databases were searched for controlled studies, resulting in 12 sMRI and $17 \mathrm{fMRI}$ studies. In line with current models on ODD/CD, studies were classified in hot and cool executive functioning (EF). Both the meta-analytic and narrative reviews showed evidence of smaller brain structures and lower brain activity in individuals with ODD/CD in mainly hot EF-related areas: bilateral amygdala, bilateral insula, right striatum, left medial/superior frontal gyrus, and left precuneus. Evidence was present in both structural and functional studies, and irrespective of the presence of ADHD comorbidity. There is strong evidence that abnormalities in the amygdala are specific for ODD/CD as compared to ADHD, and correlational studies further support the association between abnormalities in the amygdala and $\mathrm{ODD} / \mathrm{CD}$ symptoms. Besides the left precuneus, there was no evidence for abnormalities in typical cool EF related structures, such as the cerebellum and dorsolateral prefrontal cortex. Resulting areas are associated with emotion-processing, error-monitoring, problem-solving and self-control; areas associated with neurocognitive and behavioural deficits implicated in ODD/CD. Our findings confirm the involvement of
\end{abstract}

Siri D. S. Noordermeer

s.d.s.noordermeer@vu.nl

1 Faculty of Behavioural and Movement Sciences, Clinical Neuropsychology Section, VU University Amsterdam, Van der Boechorststraat 1, 1081 BT Amsterdam, The Netherlands hot, and to a smaller extent cool, EF associated brain areas in $\mathrm{ODD} / \mathrm{CD}$, and support an integrated model for ODD/CD (e.g. Blair, Development and Psychopathology, 17(3), 865-891, 2005).

Keywords ODD $\cdot$ CD $\cdot$ ADHD $\cdot$ Structural MRI $\cdot$ Functional MRI $\cdot$ ALE meta-analysis

\section{Introduction}

In recent years, magnetic resonance imaging (MRI) studies have provided insight into the underlying brain mechanisms of disruptive behaviour disorders, including oppositional defiant disorder (ODD) and conduct disorder (CD). This review will integrate and discuss studies using structural (sMRI) and functional MRI (fMRI) in these disorders. ODD and CD are developmental disorders that are among the most commonly diagnosed mental health conditions in childhood (Hamilton and Armando 2008; Loeber et al. 2009). Community samples show a prevalence rate for ODD ranging between 2 and $14 \%$ and for CD ranging between 2 and $16 \%$ (Boylan et al. 2007; Loeber et al. 2000). Both disorders are more prevalent in boys than in girls with ratio's ranging from 3:1 to 9:1 (Loeber et al. 2000). ODD is defined by a frequent and persistent pattern of irritable and angry mood, vindictiveness and developmentally inappropriate, negativistic, defiant, and disobedient behaviour toward authority figures (American Psychiatric Association 2013). CD is characterised by a persistent pattern of multiple antisocial behaviours during childhood and adolescence, including fighting, bullying, stealing, vandalism, and lying for personal gain (American Psychiatric Association 2013). Depending on whether the individual was younger or older than 10 years at the time of symptom onset, there is a 
differentiation between childhood-onset CD and adolescentonset CD (American Psychiatric Association 2013).

Although both disorders have some distinct characteristics, the general consensus is that ODD and CD are highly correlated expressions of psychopathology. Till the emergence of the DSM-5, ODD has been coined as a milder version of CD as emphasised by the hierarchical rule stipulated in the DSMIV stating that a diagnosis of ODD is precluded when CD is present, due to very high levels of ODD features in individuals with $\mathrm{CD}$ and the precursory role of ODD for $\mathrm{CD}$. This precursory role of ODD for the later development of CD is supported by a quadrupled risk for the development of $\mathrm{CD}$ in individuals with ODD (Burke et al. 2002; Loeber et al. 2009; Rowe et al. 2010). In addition to the high levels of ODD features in CD, comorbidity rates of ODD are as high as $45 \%$ in children with $\mathrm{CD}$, and in clinical samples these rates increase to up to $96 \%$ (Loeber et al. 2009; Rowe et al. 2002). ODD and CD share risk factors in both the psychosocial domain, such as poverty and social disadvantage, and the family domain, such as a history of criminality in biological parents (Rowe et al. 2002; Burke et al. 2002). An extensive review on the heritability of ODD and CD shows heritability rates of 61 and $74 \%$, respectively, with $50 \%$ of the reported genes being associated with both disorders (Coolidge et al. 2000; Lahey and Waldman 2012). The presence of either ODD or CD predicts poor future outcomes, including compromised psychiatric, family and social functioning, as well as an increased risk for adverse life events: e.g., peer rejection, criminal behaviour and incarceration at a young age (Burke et al. 2002; Hamilton and Armando 2008; Loeber et al. 2000). When ODD or CD persists, individuals are at a heightened risk for anxiety disorders and depression. Furthermore, persistence of childhood ODD or CD into adulthood results in a diagnosis of antisocial personality disorder (APD), which is in turn related to high rates of domestic violence, unemployment and homelessness (Loeber et al. 2008; Kimonis and Frick 2010). APD can only be diagnosed when there is a history of some symptoms of $\mathrm{CD}$ and the transition from CD to APD occurs in around $54 \%$ of individuals with CD (Fairchild et al. 2013b; American Psychiatric Association 2013). Treatment of ODD and CD is generally not specific to either disorder, and a combination of interventions that aim at multiple domains tends to be more successful than treating a singular domain. In the current review, ODD and CD will be treated as representing one dimension of psychopathology, because of the similarities in many domains including aetiology, phenotypical manifestation, correlated features, as well as treatment. We will refer to this dimension as ODD/CD.

Several explanatory models of ODD/CD focus specifically on neurocognitive impairments, which are thought to be related to abnormalities in underlying brain mechanisms. Neurocognitive impairments that are associated with ODD/ CD include lower IQ, deficiencies in inhibitory control, abnormalities in emotional processing and social cognition, and abnormalities in reinforcement processing. Most of the explanatory models emphasise a deficit in so-called executive functioning (EF). EF is the sum of neurocognitive processes that maintain an appropriate problem-solving set to attain a goal (Pennington and Ozonoff 1996; Willcutt et al. 2005). A well-known distinction in EF is that between hot and cool EF. Hot EF is characterised by motivational and affective aspects of cognitive processing, such as reinforcement learning, affective decision-making and emotional processing (Anderson et al. 2008; Blair and Lee 2013; Kerr and Zelazo 2004; Zelazo and Carlson 2012). Brain areas that are reported to be important for hot EF include the amygdala, anterior cingulate cortex, insula and orbitofrontal cortex (Crowe and Blair 2008; Prencipe et al. 2011; Rubia 2011). In contrast, cool EF refers to goal-directed and problem-solving behaviours, as well as self-regulation, not involving motivational or affective aspects. Cool EF encompasses functions using diverse abilities such as inhibition, working memory, planning, flexibility, and the ability to creatively generate solutions for problems (Sarkar et al. 2013; Diamond 2013). Brain areas reported to be central to cool EF include the dorsolateral prefrontal cortex and the cerebellum (Prencipe et al. 2011; Rubia 2011; Yang and Raine 2009; Sterzer and Stadler 2009). This distinction between hot and cool EF provides a framework to study underlying brain mechanisms of observed behavioural and neurocognitive abnormalities in ODD/CD. This knowledge can help test theoretical models on ODD/CD through clarifying involvement of brain areas central to those models. This important information on theoretical model building can in turn help to further advance the field, by yielding supporting or opposing evidence for the involvement of brain areas.

An altered reinforcement system, and thus a hot EF problem, was proposed in early models on ODD/CD by Quay (1965, 1993) and by Newman and Kosson (1986). Indeed, a recent extensive review in antisocial individuals showed altered sensitivity to reward and punishment and processing of these contingencies (Byrd et al. 2014). Specifically, antisocial individuals show an increased affinity for immediate reward over delayed reward, an insensitivity to punishment, and increased rewardseeking (Byrd et al. 2014). This altered reinforcement sensitivity has been related to problems in social cognition in individuals with ODD/CD. For example, these individuals tend to show a preference for more aggressive reactions in social situations, which might be due to their unsparing surge for reward and decreased punishment sensitivity (Burke et al. 2002; Loeber et al. 2008; Quay and Hogan 1999; Rubia 2011). In addition, abnormalities in emotional processing have been reported repeatedly in studies with ODD/CD samples (Byrd et al. 2014), including reduced levels of empathy and deficits in the recognition of emotional expressions (Blair 2013). Both abnormalities in reinforcement sensitivity and emotional processing in $\mathrm{ODD} / \mathrm{CD}$ have been related to abnormalities in hot $\mathrm{EF}$ brain 
areas, such as the amygdala and the striatum (Crowe and Blair 2008; Prencipe et al. 2011).

Another important explanatory model, that is more fitting with a cool EF deficit, was proposed by Moffitt (1993), who distinguished between adolescent-limited $\mathrm{ODD} / \mathrm{CD}$, and the more severe, life-course persistent ODD/CD (Moffitt 1993). According to that model, adolescent-limited ODD/CD is merely a stage in development during which adaptive social behaviour is tested and learned, while life-course persistent $\mathrm{ODD} / \mathrm{CD}$ is thought to arise of an interplay between a difficult and under-controlled temperament and adverse environmental factors. This under-controlled temperament is thought to be promoted by a deficit in cool EF, including difficulties in inhibition and self-control (Burke et al. 2002; Loeber et al. 2008; Moffitt 1993). Evidence from neurocognitive studies generally points toward a range of abnormalities in cool EF in ODD/CD, such as low IQ, inefficiencies in problem solving, and less than optimal inhibitory control (Oosterlaan et al. 1998; Burke et al. 2002; Loeber et al. 2008; Quay and Hogan 1999, but see Van Goozen et al. 2004), and have been related to abnormalities in the dorsolateral prefrontal cortex and cerebellum (Yang and Raine 2009; Prencipe et al. 2011).

Finally, one of the most influential explanatory models at present is proposed by Blair, who suggests that individuals with ODD/CD demonstrate impairments in two separate circuits associated with hot and cool EF (Blair 2005). According to Blair (2005), the first compromised circuit is involved in emotional processing and regulation and is responsible for an increase in antisocial behaviour. The key component of brain areas underlying this mainly hot EF circuit is supposedly the amygdala. The second compromised circuit is involved in response inhibition and is responsible for loss of temper and exaggerated aggressive responses in individuals with ODD/ $\mathrm{CD}$. The key component of brain areas underlying this mainly cool EF circuit is supposedly the ventrolateral frontal cortex.

Support for the explanatory models on ODD/CD is well established in behavioural studies. However, knowledge about these models in terms of structural and functional neuroimaging is incomplete. While a review of the current structural and functional neuroimaging literature would be an ideal way to provide neurobiological evidence to confirm or reject a model, such a review has not been conducted so far. The current review aims to fill this gap with the goal to enhance insight into the underlying mechanisms of ODD/CD and additionally test the plausibility of hot and cool EF models in $\mathrm{ODD} / \mathrm{CD}$ in terms of neural mechanisms.

A highly comorbid condition of ODD/CD is attentiondeficit hyperactivity disorder (ADHD), one of the most commonly diagnosed disruptive behaviour disorders in children. The percentage of individuals diagnosed with ODD/CD that additionally qualify for a comorbid ADHD diagnosis ranges up to $35 \%$, and up to $50 \%$ of children with disruptive behaviours show symptoms of both ADHD and ODD/CD (Loeber et al. 2000; Anderson and Kiehl 2013; Connor et al. 2010; Waschbusch 2002). In children and adolescents with ODD/ $\mathrm{CD}$ and comorbid ADHD, the prognosis, including the risk to develop anxiety and depressive disorders and antisocial personality disorder, is considerably worse than when only ODD/ CD or only ADHD is present (Dolan and Lennox 2013; Loeber et al. 2000). In addition, this comorbid group shows an earlier age of symptom onset, exhibits more physical aggression and delinquency, shows significantly higher ODD, $\mathrm{CD}$ and ADHD symptom severity, and shows more functional impairments than a group with any of these diagnoses in singularity (Anderson and Kiehl 2012; Loeber et al. 2000; Waschbusch 2002). This highlights the importance of clarifying the specificity of abnormalities associated with ODD/CD and contrasting ODD/CD-only with ODD/CD+ADHD, when studying these disorders.

Although previous reviews have addressed structural and functional brain abnormalities in ODD/CD (Matthys et al. 2013; Rubia 2011), these reviews were neither systematic reviews, nor focussed exclusively on diagnostic groups of $\mathrm{ODD} / \mathrm{CD}$, nor were set out to investigate the relative contribution of ADHD. To address structural and functional brain anatomical aspects of $\mathrm{ODD} / \mathrm{CD}$, the current comprehensive review includes a complete systematic narrative review as well as meta-analyses using ALE (Eickhoff et al. 2009, 2012; Laird et al. 2005) of the available structural and functional imaging studies. The quantitative approach increases the precision and the power of reported results compared to a purely qualitative review, and indicates which brain areas are most robustly implicated in $\mathrm{ODD} / \mathrm{CD}$. However, since an ALE meta-analysis does not allow inclusion of studies reporting on non-significant group differences, the narrative review provides the necessary balance in interpreting the findings. The narrative review complied with the standards of a systematic review, performing a literature search based on a detailed plan and search strategy, and had the goal of reducing bias by identifying, appraising and synthesising all relevant studies on this topic. In addition, we investigated the specificity of brain correlates. Firstly, we investigated specificity by discussing results from studies using ODD/CD-only samples separately from results from studies using ODD/CD+ADHD samples. Secondly, we compared results of samples including individuals with $\mathrm{ODD} / \mathrm{CD}$ (with and without comorbid ADHD) to samples including individuals with ADHD-only. In addition, we reported associations between abnormalities in investigated structures and ODD/CD related symptoms. Knowledge about the specificity of reported abnormalities may help to clarify the heterogeneity in studies on ODD/CD.

This review is divided in two sections. The first section deals with structural findings, integrating findings for all reported brain areas; the second section deals with functional findings, and is divided in a part describing hot EF and a subsequent part describing cool EF, based on the assessed 
tasks and contrasts. Each section starts with a quantitative meta-analysis, for which an activation likelihood estimation (ALE) meta-analysis was performed. ALE is a technique that is used to identify significant anatomical locations for which effects are consistent, and that is robust to publication bias (for a detailed description of ALE see: Eickhoff et al. 2009; Laird et al. 2005; Eickhoff et al. 2012). This quantitative metaanalysis is followed by a narrative review. Then, both the sections on structural and functional findings are integrated to provide a complete overview of all involved brain areas and to assess the evidence for abnormalities in terms of hot and cool EF related brain areas. We expected to find abnormalities in brain structure and function of individuals with ODD/CD subserving both hot EF (i.e., the amygdala, anterior cingulate cortex, insula, and orbitofrontal cortex (Rubia 2011; Prencipe et al. 2011; Crowe and Blair 2008)) and cool EF (i.e. the dorsolateral prefrontal cortex and cerebellum (Rubia 2011; Prencipe et al. 2011; Yang and Raine 2009; Sterzer and Stadler 2009)), which would be in line with an integrated model such as the model proposed by Blair.

\section{Methods}

\section{Study Selection}

This review included all empirical studies that met the following inclusion criteria: (1) the study reported on functional or structural magnetic resonance imaging results, comparing (a) individuals with ODD/CD with or without comorbid ADHD to control subjects, and if included, to individuals with ADHD-only, or (b) individuals with ODD/CD without ADHD to ODD/CD individuals with ADHD. The control group of each study was carefully checked on reported psychiatric disorders, and when a study reported on the presence of any psychiatric disorder in participants of the control group, that study was excluded from the current study. This resulted in the exclusion of two studies. (2) Diagnosis of the participants had to be based on DSM-III, DSM-IV or DSM-5 criteria. (3) The study had to be published in a peer-reviewed English language journal. No limits were set on the ages of participants. All relevant studies published up till June 2015 were incorporated.

The databases PubMed, EMBASE, PsycInfo and Web of Science were searched, using the search terms ODD, CD, disruptive behavioural disorder, disruptive behaviour, externalising behavioural disorder, externalising behaviour, MRI, neuroimaging, and equivalent MeSH terms. Furthermore, reference lists of selected studies and reviews were checked for additional relevant studies. A total of 576 studies were initially retrieved and screened, after which a total of 67 studies remained that fulfilled inclusion criteria based on screening of the title and abstract. These 67 studies were further assessed for eligibility using the full text of the study, resulting in 29 studies that met inclusion criteria and were incorporated in the present review; see Fig. 1 for the flow diagram of included studies. The 29 studies selected for review included a total of 1278 individuals, including 713 patients and 565 controls.

In this review, we distinguished the following samples: (1) ODD/CD-only, including only individuals with ODD/CD without comorbid ADHD, (2) ODD/CD-mixed, including both individuals with ODD/CD-only and individuals with $\mathrm{ODD} / \mathrm{CD}+\mathrm{ADHD}$, and (3) ODD/CD+ADHD, including only individuals classifying for both $\mathrm{ODD} / \mathrm{CD}$ and ADHD. We included a total of 10 ODD/CD-only studies (7 structural, 3 functional), 14 ODD/CD-mixed studies (9 structural, 5 functional), and $5 \mathrm{ODD} / \mathrm{CD}+\mathrm{ADHD}$ studies (1 structural, 4 functional). By differentiating between these three samples, it was possible to discern whether an abnormality was related to ODD/CD and not confounded by comorbid ADHD, which would be suggested when an abnormality is found in an ODD/CD-only sample. Alternatively, when an abnormality is found in an ODD/CD-mixed or ODD/CD+ADHD sample, it may be possible that the abnormality is also related to comorbid ADHD. Therefore, results are discussed separately for ODD/CD-only samples versus controls and for ODD/CDmixed and ODD/CD+ADHD samples versus controls. When included in a study, results from additional comparisons with an ADHD-only sample were provided.

The section on structural neuroimaging findings reports all brain areas, not distinguishing between hot and cool EF. The section on functional neuroimaging findings is divided in two parts: the first part is focused on hot EF and the second part is focused on cool EF. Classification of studies as hot or cool was done in line with the literature. Two authors independently inspected the task characteristics and the reported contrasts for each study and judged these as assessing either hot or cool EF. In case of disagreement, the third author was consulted, resulting in full agreement on all studies. One study (Rubia et al. 2009b) targeted both hot and cool EF and we therefore discussed this study in both parts of the fMRI results. After presentation of the meta-analytic and narrative results of the structural and functional studies, findings are integrated for structural and functional studies in order to provide an overview of all relevant literature and resulting conclusions in terms of hot and cool EF related brain areas.

\section{Meta-analysis: Activation Likelihood Estimation}

For the quantitative meta-analysis, an activation likelihood estimation (ALE) meta-analysis was performed using the Brainmap GingerALE software package (Eickhoff et al. 2009, 2012; Laird et al. 2005). For the ALE meta-analysis to be reliable, the minimum number of studies to be included is five (www.brainmap.org). The algorithm used by GingerALE 
Fig. 1 PRISMA flow diagram showing the process of study selection

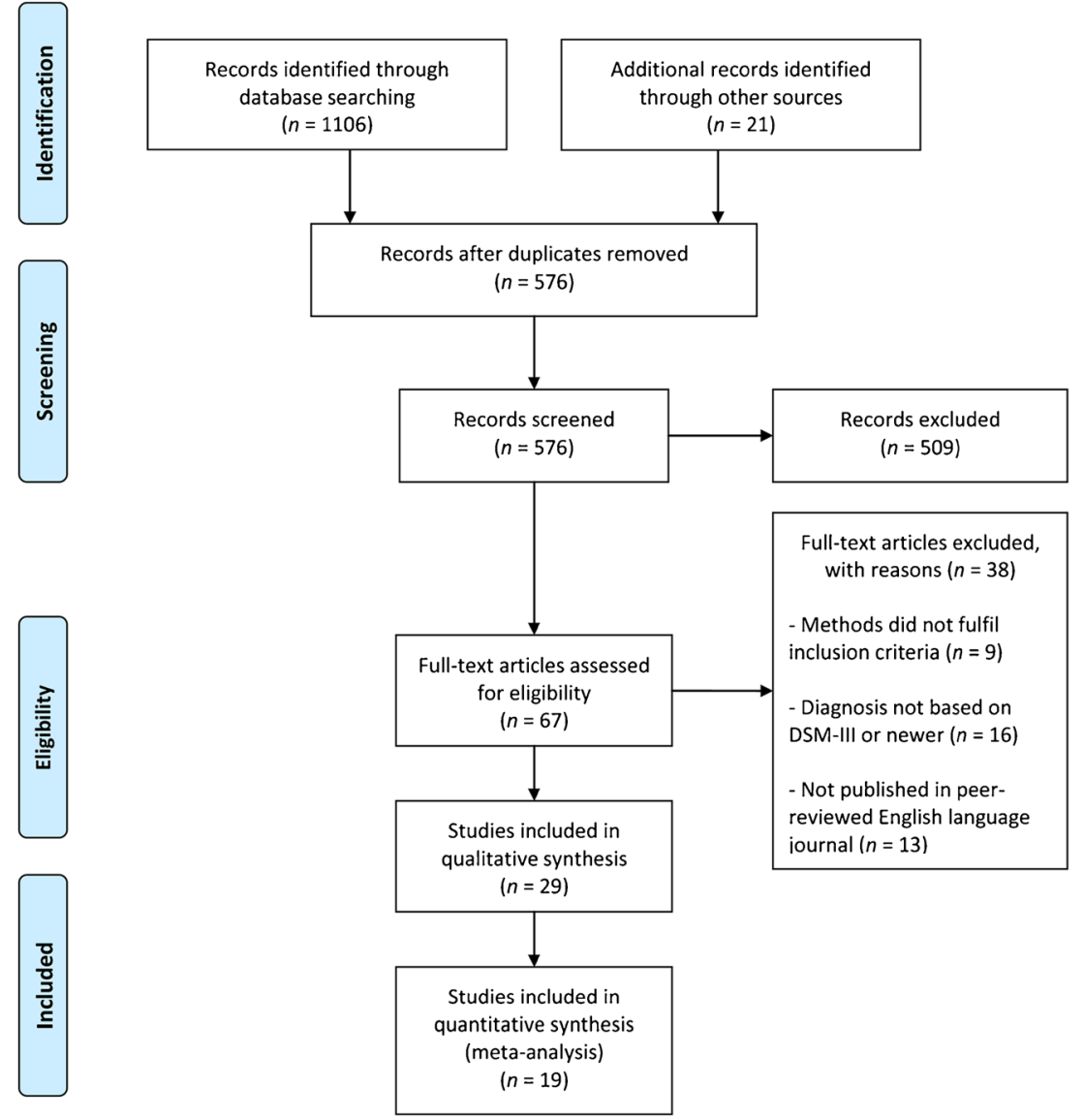

applies a random-effects approach to identify anatomical locations for which effects are observed most consistently, and which renders it robust for the possible effects of publication bias (Fox et al. 1998). Analyses were performed separately for the structural and the functional studies, and for the functional studies these were performed separately for studies on hot and on cool EF. Studies eligible for inclusion in the meta-analysis were additionally required to report $\mathrm{x} / \mathrm{y} / \mathrm{z}$ coordinates for clusters showing group differences in volume (structural MRI) or activity (functional MRI) in either Montreal Neurological Institute (MNI) or Talairach space.

All coordinates originally reported in MNI space were normalised to Talairach space using Lancaster's Transform; coordinates which were already in Talairach space were converted back to MNI coordinates and subsequently normalised to Talairach space using Lancaster's Transform, to account for divergent analyses procedures and minimise differences in coordinates between studies (Lancaster et al. 2007; Laird et al. 2010). The 2.3.1 version of GingerALE (Eickhoff et al. 2009) was applied in the current study and used the coordinates of the reported voxels of each study, referring to the areas that showed a group difference, as a probability distribution to create an ALE distribution map (Turkeltaub et al. 2002; Eickhoff et al. 2009, 2012). Results across studies were aggregated by GingerALE, and modelled activation maps were generated by calculating the probability that a particular voxel was activated as the union of probabilities for that voxel across studies. The contribution of each study to the metaanalytic result was weighted using the study's sample size, by widening the Gaussian distribution for a voxel with smaller samples to compensate for spatial uncertainty. Meta-analytic maps were generated by combining all the modelled activations maps, and were subsequently corrected for multiple comparisons using Family Wise Error correction. This ensured that differences between studies in terms of the number of areas showing significant group differences (e.g., due to applying a lower statistical threshold), did not influence the combined ALE map (Turkeltaub et al. 2012). This combined ALE map was then compared to a map from a null distribution with the same number of foci, but now randomly placed throughout the grey matter of the brain. After this, the final ALE map was thresholded at $p<.05$ using a False Discovery Rate (FDR) correction for multiple comparisons, and a minimum cluster size of $100 \mathrm{~mm}^{3}$ (see www.brainmap.org/ale/).

Both studies using a region of interest (ROI) approach, thus a hypothesis driven pre-selection of specific brain areas, and studies using a whole brain analyses (WBA) approach, thus studying the entire brain, were included in this review. To test the possibility that studies using an ROI approach might bias meta-analytic results due to less stringent statistical criteria, 
the resulting map of all studies (using both WBA and ROI approach) was compared to the resulting map from studies with a WBA approach. When differences between the maps were present, results from the map including only the WBA studies were reported. The ALE map was overlaid onto a Talairach anatomical template for visualisation purposes, and the areas reported in our Results section refer to the locations of the extrema.

\section{Results}

Results of the reviewed studies are discussed in two main sections with the first section summarising the structural neuroimaging findings and the second section summarising the functional neuroimaging findings. Both sections start with a general overview of all included studies, after which the results from the meta-analysis are reported, followed by the results from the narrative review. The narrative parts contain a summary of the main findings for ODD/CD-only groups first since those are not biased by comorbid ADHD, followed by the main findings in ODD/CD-mixed and ODD/CD+ ADHD groups, and ends with the correlational findings and the specificity of findings regarding ODD/CD.

After presentation of the meta-analytic and narrative results of the structural and functional studies, structural and functional findings are integrated in order to provide an overview of all relevant literature and resulting conclusions in terms of hot and cool EF related brain areas. Since an ALE metaanalysis cannot include studies that reported on nonsignificant group differences, but rather identifies anatomical locations for which effects are observed most consistently, findings from the ALE meta-analysis are combined with the results of the narrative review to provide a comprehensive and balanced overview of available evidence.

\section{Structural Neuroimaging Findings}

General Overview Table 1 shows a total of 12 studies that investigated structural differences in individuals with ODD/ CD-only (three studies), ODD/CD-mixed (five studies), or $\mathrm{ODD} / \mathrm{CD}+\mathrm{ADHD}$ (four studies) compared to controls. All 12 studies used WBA to investigate regional brain differences, while three studies additionally used an ROI approach (Fairchild et al. 2011, 2013a; Sterzer et al. 2007). Two of these studies also reported on results of contrasts between ODD/CD (with and without comorbid ADHD) and ADHD-only. In addition, seven studies investigated the association between brain region volumes and symptom counts of ODD/CD (Sterzer et al. 2007; Huebner et al. 2008; Fahim et al. 2011; Fairchild et al. 2011, 2013a; Stevens and Haney-Caron 2012; Michalska et al. 2015).
All studies investigated children/adolescents. For information regarding group and study characteristics of the included studies, see Table 1. Reported differences in brain structures are bilateral, unless reported to be either left or right sided. Results are reported from WBA approaches, unless specified to be the result of ROI based analyses, see Table 1 .

Structural ALE Meta-analysis Eight structural studies, all using a WBA approach, reported coordinates and were included in the meta-analysis (studies marked with ${ }^{\mathrm{a}}$ in Table 1). Due to the limited number of studies in ODD/CD-only (three studies) it was not possible to perform an ALE meta-analysis on studies comparing ODD/CD-only groups and controls (minimum number of studies required is five). Hence, the ALE meta-analysis was performed on all ODD/CD samples, therewith including both individuals with and without comorbid ADHD. Total sample sizes for all included studies ranged between 24 and 90 individuals, adding up to a total of 415 individuals, of which 267 were patients and 148 were controls (age range 8-21 years). Five studies used a full male sample, two studies used a sample consisting of $71 \%$ males and one study used a full female sample. These studies provided a total of 58 foci of grey matter volume abnormalities in individuals with ODD/CD with and without comorbid ADHD.

The ALE analysis revealed four significant clusters of altered grey matter volumes that differed between patients and controls (see Fig. 2 for visualisation). The largest cluster $\left(760 \mathrm{~mm}^{3}\right)$ was located in the left amygdala, with three foci inside this cluster. A second cluster $\left(456 \mathrm{~mm}^{3}\right)$ was found in the left insula, containing three foci. A third cluster $\left(352 \mathrm{~mm}^{3}\right)$ was located in the left medial/superior frontal gyrus, containing two foci. The fourth cluster $\left(216 \mathrm{~mm}^{3}\right)$ was located in the right insula, containing two foci.

Narrative Review Table 1 provides an overview of investigated samples and approach (WBA or ROI) and summarizes the findings of the 12 studies reporting on sMRI. Total sample sizes for all included studies ranged between 20 and 111 individuals, adding up to a total of 643 individuals, including 334 patients and 309 controls (age range 6-21 years). The samples consisted predominantly of males, with five studies using a full male sample, six studies using a sample largely consisting of males (ranging from 48 to $90 \%$ ), and one study using a full female sample.

$O D D / C D$-only versus controls (3 studies). For total grey matter volume, one of three studies reported a reduction in the $\mathrm{ODD} / \mathrm{CD}$-only group. In terms of regional grey matter volumes, abnormalities in the insula (left and bilateral), amygdala (left and bilateral), the cingulate cortex (left and paracentral), the inferior frontal gyrus (left and right), and the left dorsomedial prefrontal cortex were repetitively reported (two out of three studies). 


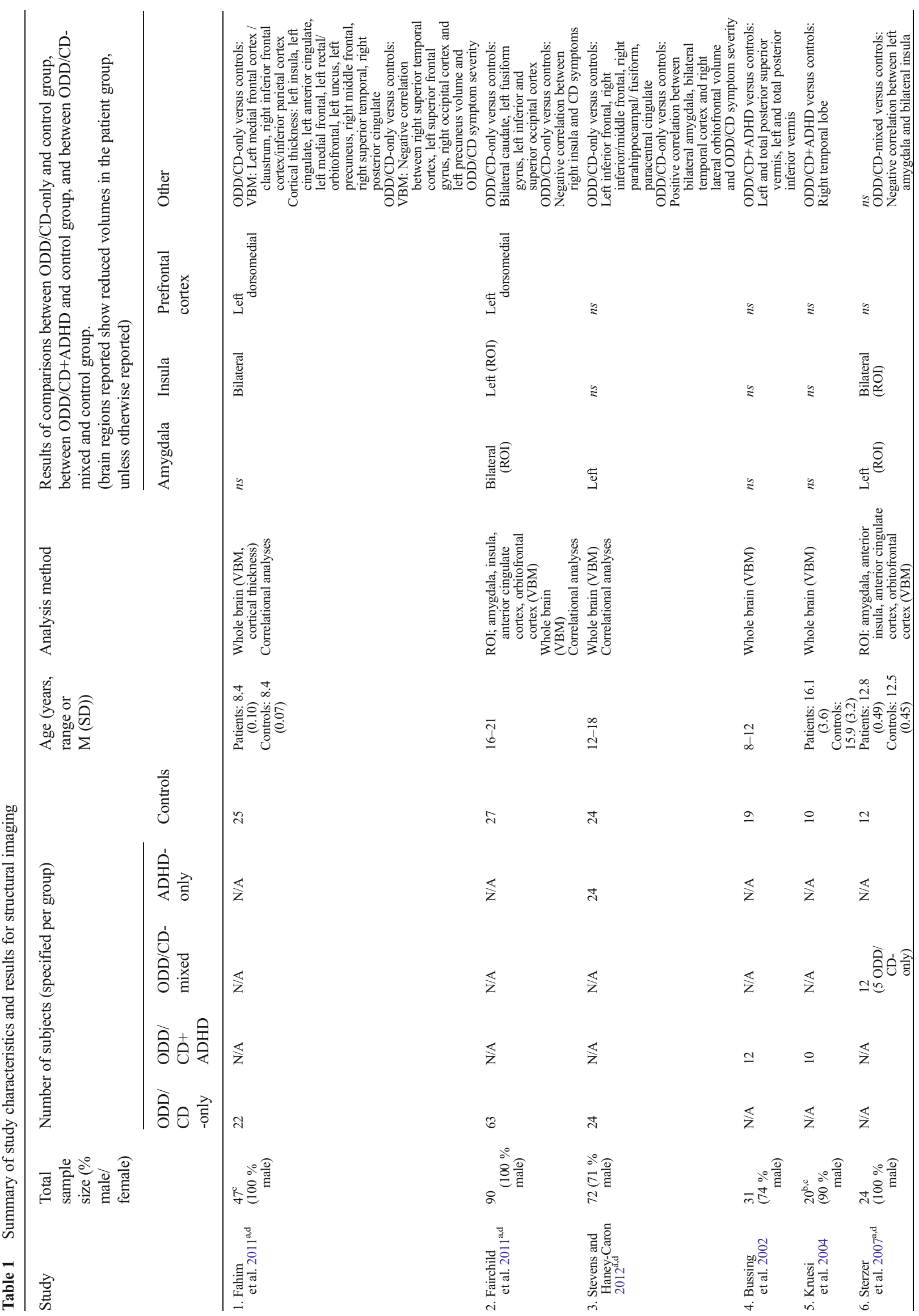




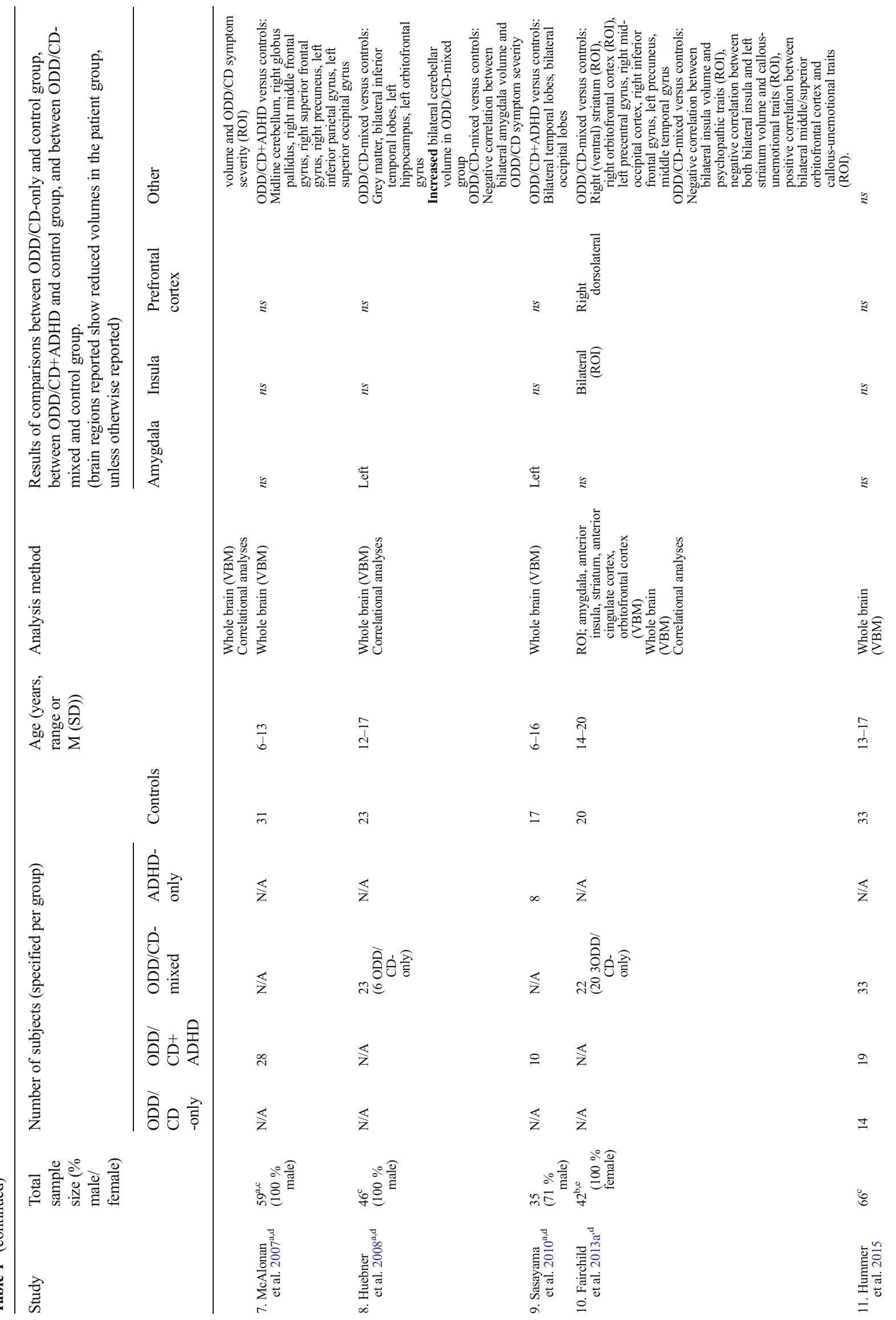




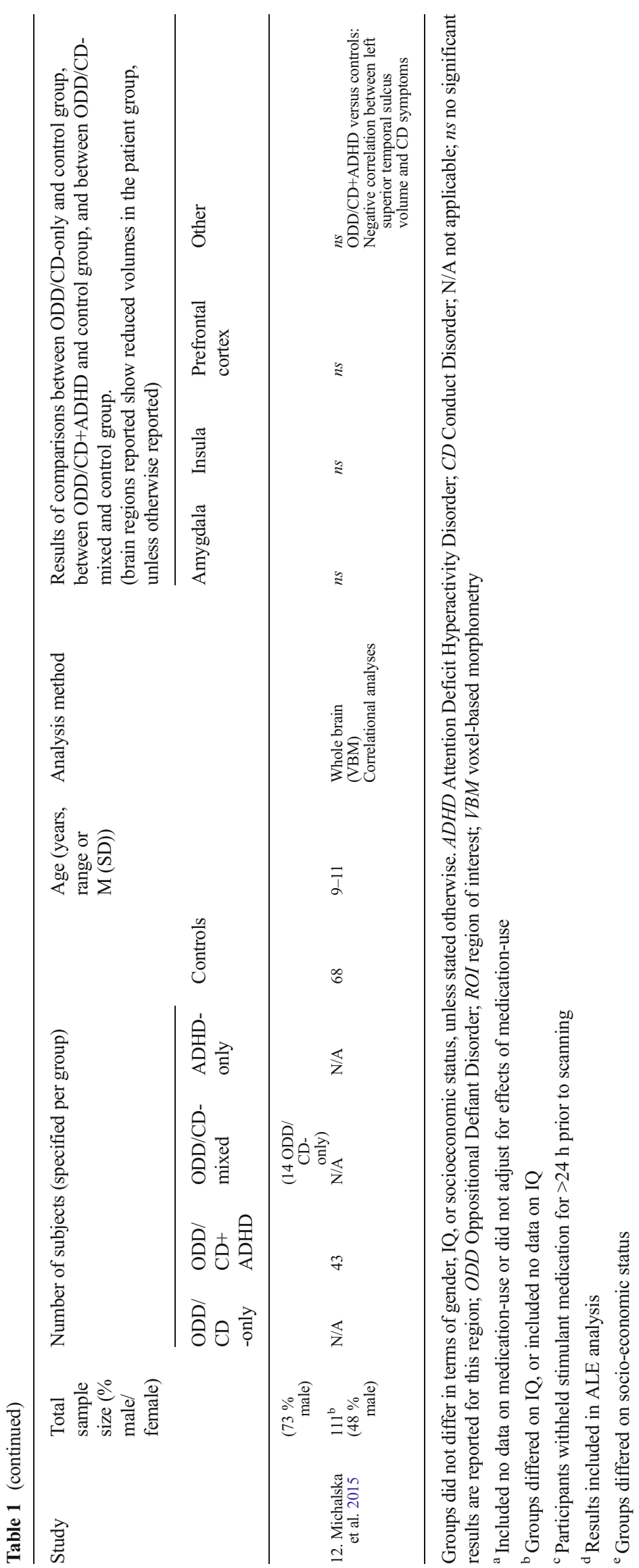


Fig. 2 Results of the structural ALE meta-analysis showing the clusters in left amygdala, insula, and left medial/superior frontal gyrus $\left(p_{\text {corrected }}<0.05\right)$ superimposed on a structural scan in Talairach space. Top row: coronal view, bottom row: axial view. $L$ Left, $R$ Right

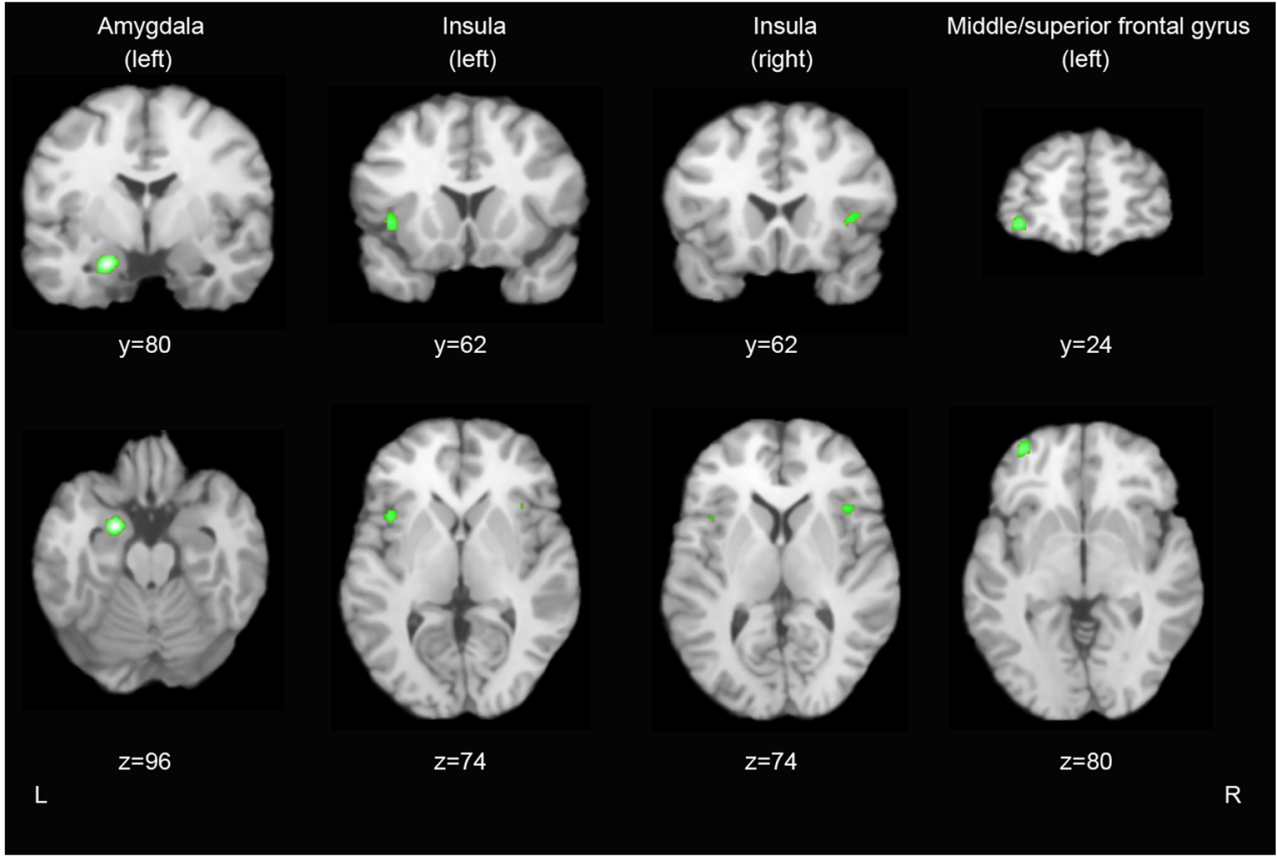

$O D D / C D$-mixed and $O D D / C D+A D H D$ (9 studies). Two of the four studies investigating total grey matter in ODD/CDmixed and ODD/CD+ADHD samples reported smaller volumes in the diagnostic groups, compared to controls. In accordance with results from the studies in the ODD/CD-only group, three of the nine studies in ODD/CD-mixed and ODD/ $\mathrm{CD}+\mathrm{ADHD}$ groups provided support for abnormalities in the left amygdala. Additionally, abnormal volumes were reported in the ODD/CD-mixed and ODD/CD+ADHD groups for structures that were not found for ODD/CD-only groups, which might indicate that these abnormalities reflect the presence of comorbid ADHD rather than ODD/CD. These structures with smaller volumes were the temporal lobe (four out of nine studies: one left, one right, two bilateral) and the cerebellum (three out of nine studies: one left, two bilateral).

Correlational findings and specificity. A total of seven studies investigated relations between grey matter abnormalities and ODD/CD symptoms. Three out of seven studies reported associations for the amygdala, insula and temporal cortex. The three studies that reported associations between the amygdala and ODD/CD symptoms, showed either a positive association (thus a larger volume related to more $\mathrm{ODD} / \mathrm{CD}$ symptoms) for the amygdala in an ODD/CD-only sample, or negative associations (thus a smaller volume related to more ODD/CD symptoms) for the bilateral or left amygdala in ODD/CD-mixed samples. For the insula, all three studies reported negative associations, one between the right insula and $\mathrm{ODD} / \mathrm{CD}$ symptoms in an ODD/CD-only sample and two between the insula and ODD/CD symptoms in ODD/CDmixed samples. For the three studies that found associations between the superior temporal cortex and ODD/CD symptoms, one study reported a positive association for the superior temporal cortex in an ODD/CD-only sample, while the other two studies reported a negative association for the right superior temporal cortex in an $\mathrm{ODD} / \mathrm{CD}$-only sample and for the left superior temporal cortex in an $\mathrm{ODD} / \mathrm{CD}+\mathrm{ADHD}$ sample.

In terms of specificity of abnormalities for ODD/CD as compared to ADHD, one study reported grey matter reductions in the amygdala and frontal gyrus to be specific for ODD/CD-only, since these were not present in the ADHDonly group. In addition, another study looked at disorder specificity by covarying for $\mathrm{ODD} / \mathrm{CD}$ in an $\mathrm{ODD} / \mathrm{CD}$-mixed group and reported that the grey matter reductions in the amygdala turned non-significant, suggesting that these grey matter reductions were specific for ODD/CD.

Structural Summary Combining the results from the metaanalysis and the narrative review, structural neuroimaging studies most consistently implicated abnormalities of the left amygdala, insula and left frontal gyrus in ODD/CD.

\section{Functional Neuroimaging Findings}

General Overview Tables 2 and 3 shows a total of 17 studies that investigated brain activity correlates in individuals with ODD/CD-only (seven studies), ODD/CD-mixed (nine studies), or ODD/CD+ADHD (one study) compared with typically developing individuals. Of the 17 included studies, 14 conducted a WBA approach to investigate regional brain differences, of which 5 also conducted ROI analyses (S.F. White et al. 2013; Finger et al. 2011; Herpertz et al. 2008; Passamonti et al. 2010; Cohn et al. 2014). In three studies only ROI analyses were reported (Gatzke-Kopp et al. 2009; Marsh et al. 2008; Sterzer et al. 2005). Seven studies investigated the association between 


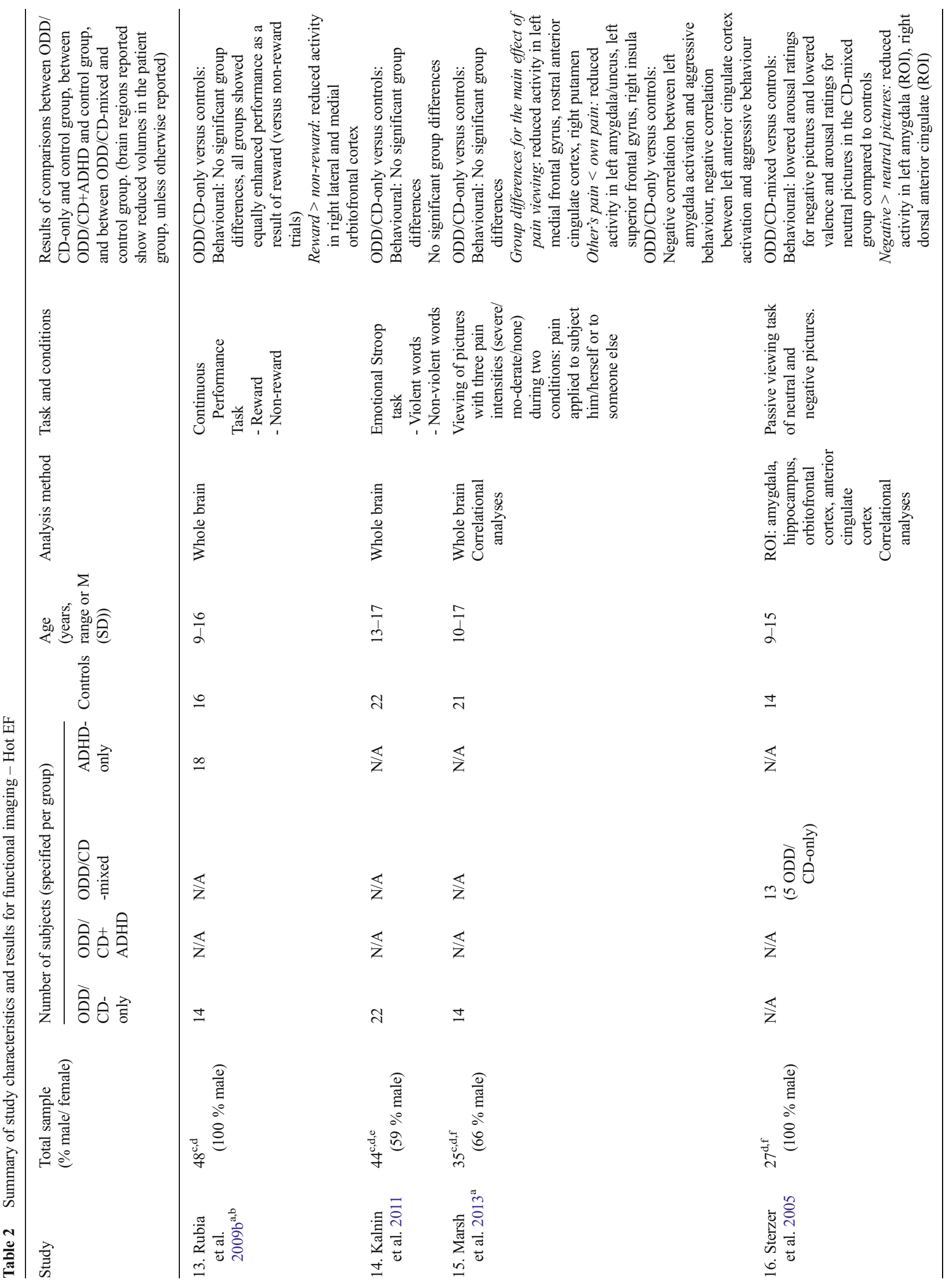




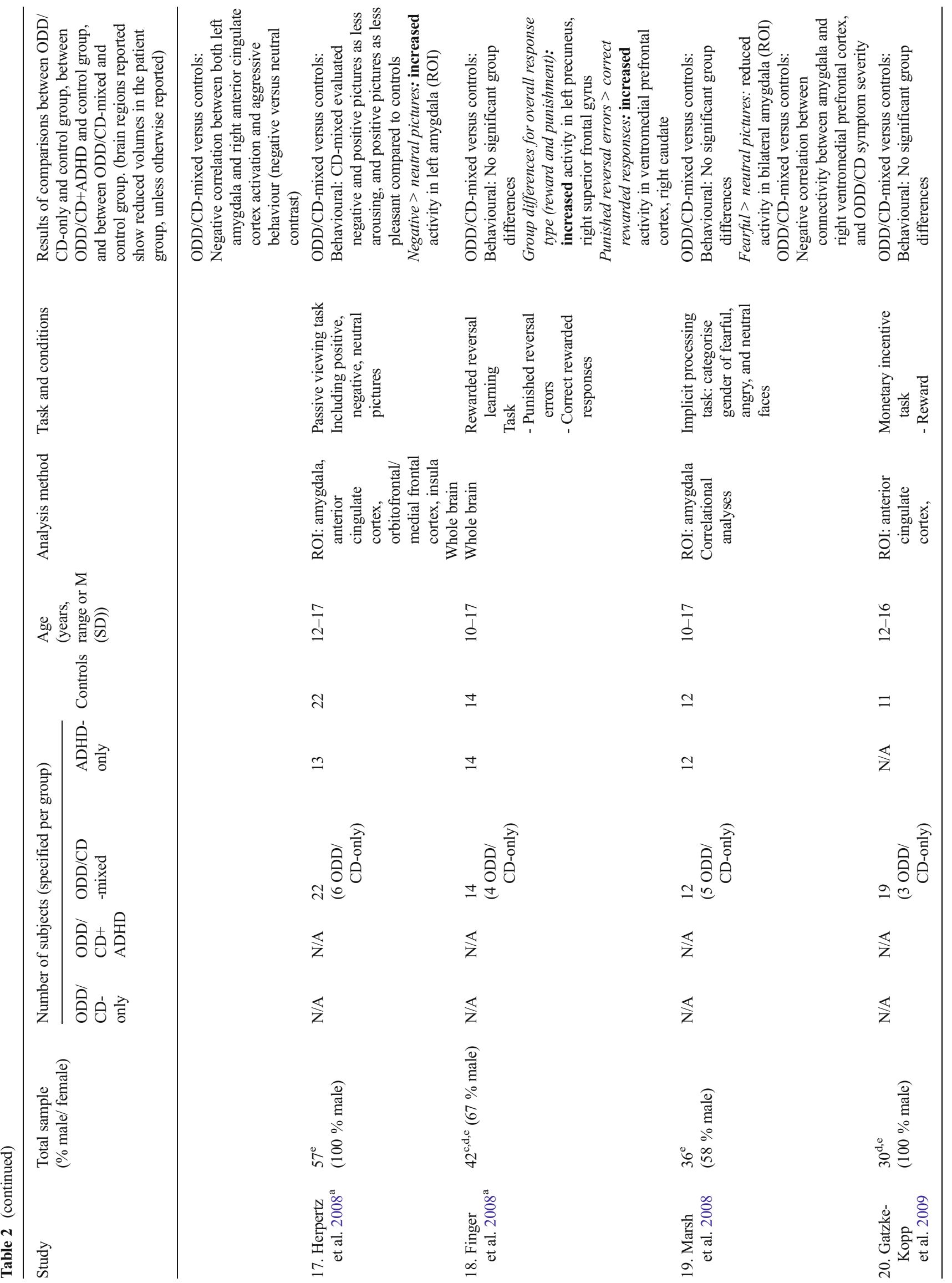




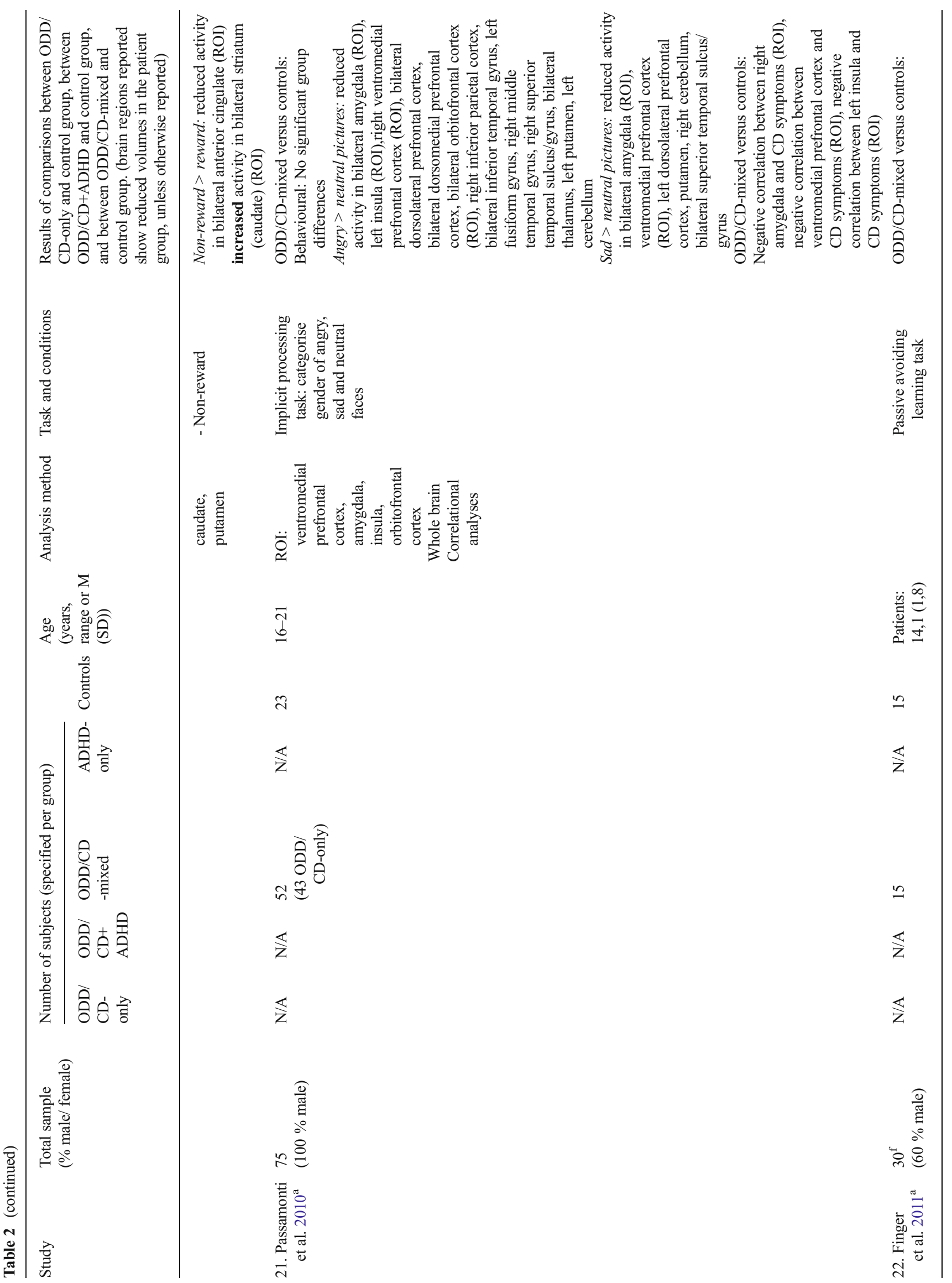




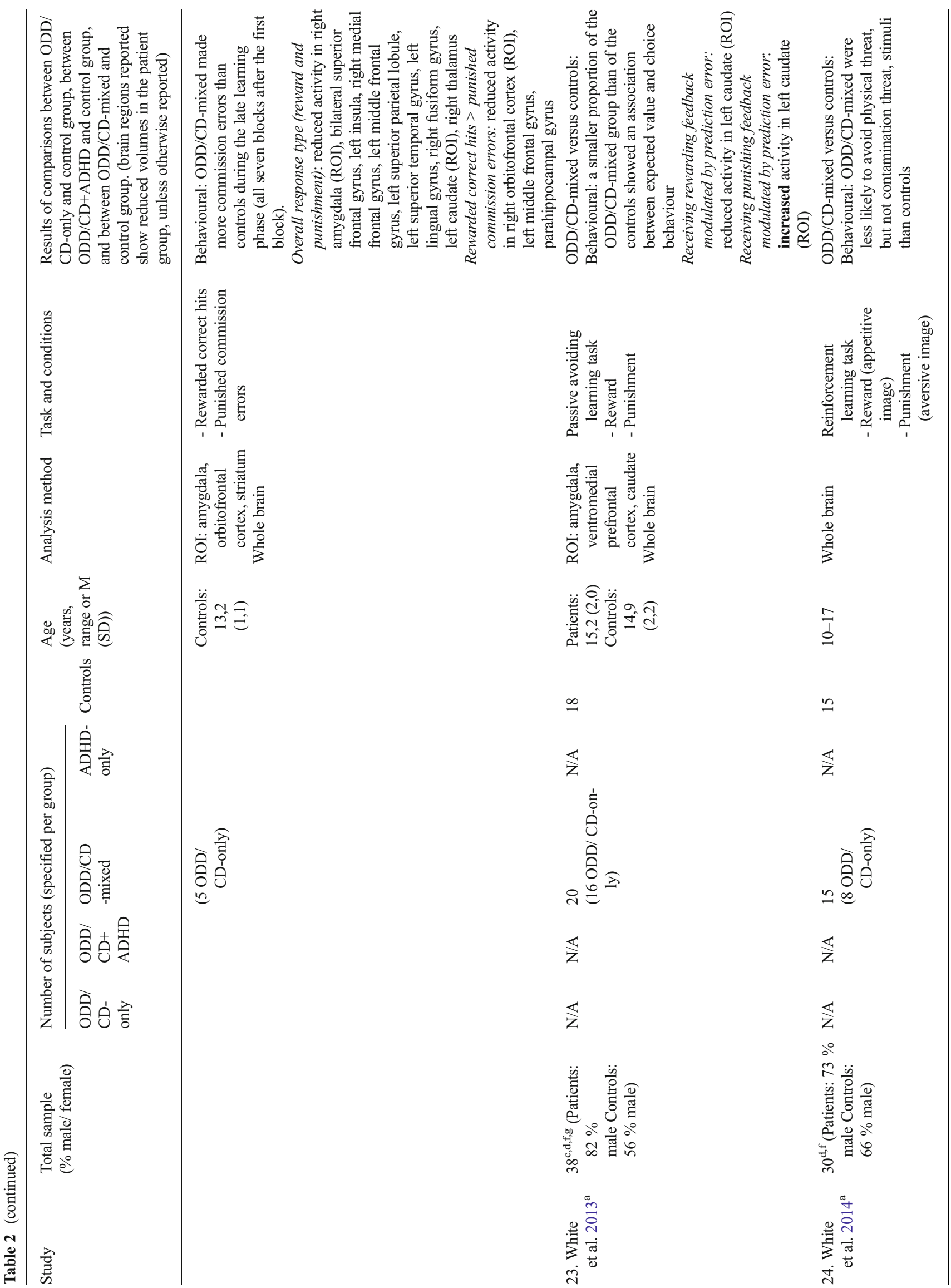




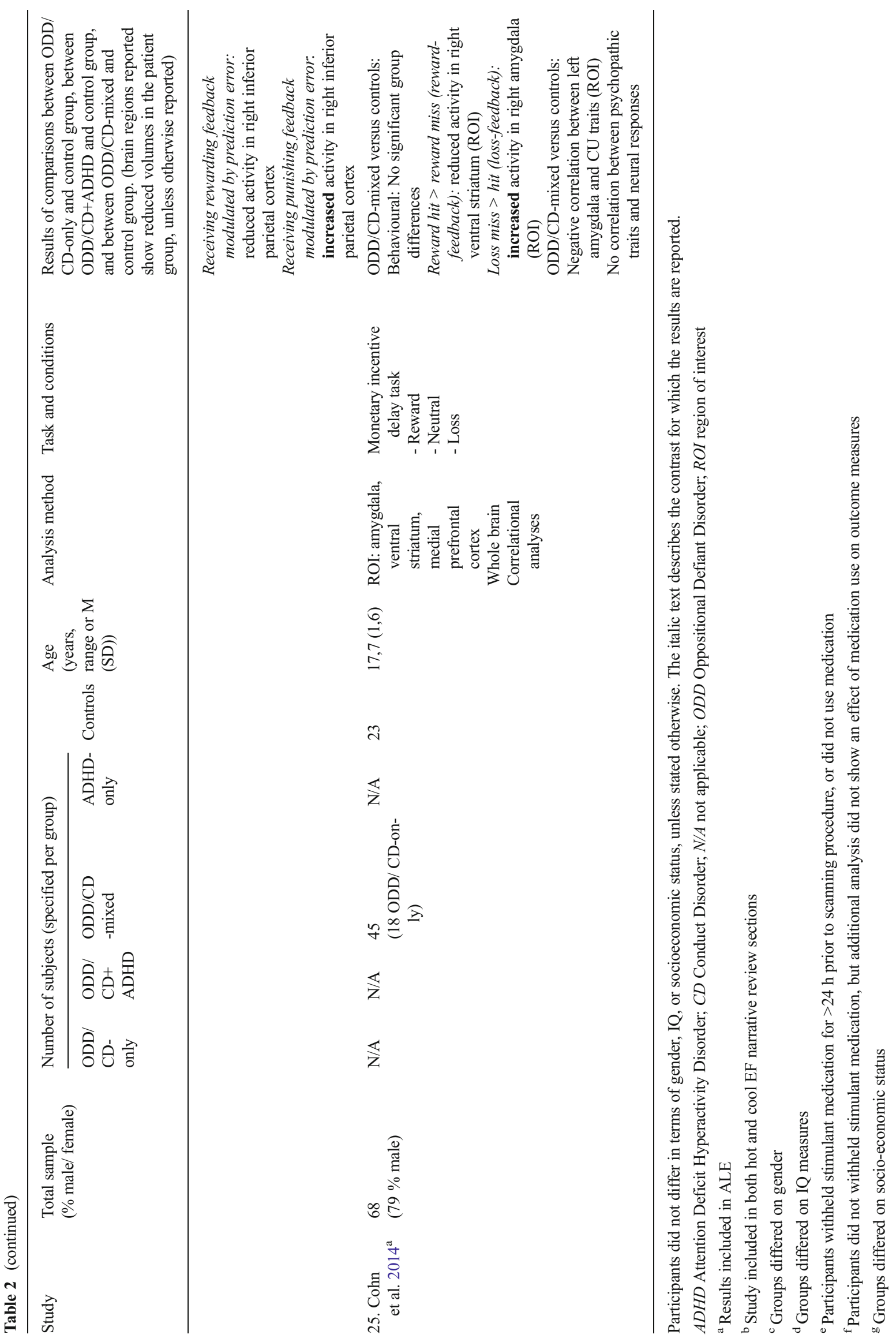




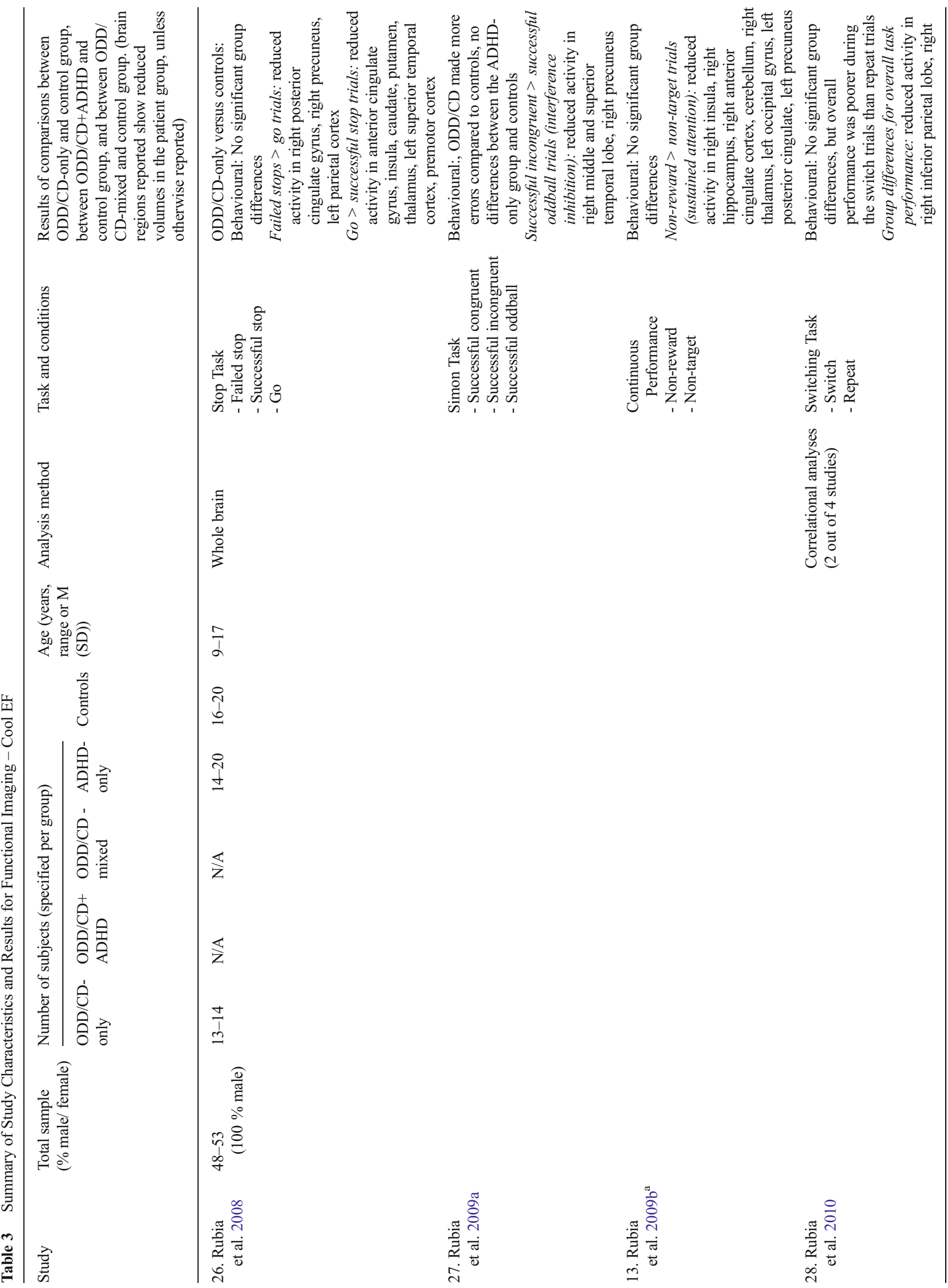




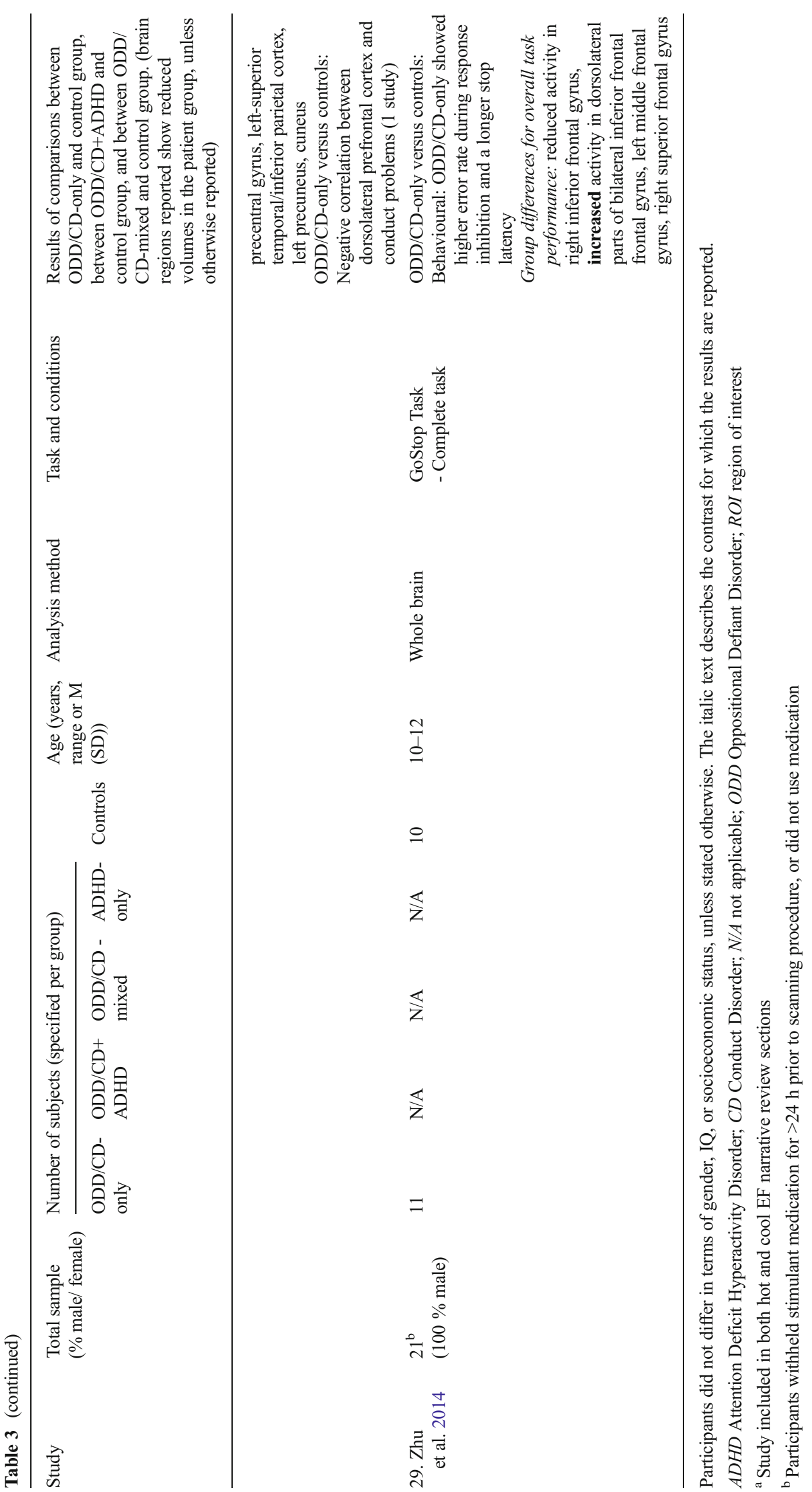


activity in brain areas and ODD/CD symptom counts (A.A. Marsh et al. 2013; Sterzer et al. 2005; Passamonti et al. 2010; Cohn et al. 2014; Finger et al. 2008; Rubia et al. 2008, 2010).

We reviewed the studies using the framework of hot and cool $\mathrm{EF}$ in $\mathrm{ODD} / \mathrm{CD}$, which resulted in 13 studies in the hot EF section and 5 studies in the cool EF section, of which 1 study appeared in both sections (Rubia et al. 2009b). The 13 studies in the hot EF section included groups with ODD/CD-only (three studies), ODD/CD-mixed (nine studies) or ODD/CD+ADHD (one study). In all studies, these groups were compared with a typically developing group and in four studies these groups were additionally compared with an ADHD-only group (Finger et al. 2008; Herpertz et al. 2008; Marsh et al. 2008; Rubia et al. 2009b). The five studies in the cool EF section only included individuals with ODD/CD-only that were compared to a typically developing group and in four of the studies the ODD/ CD-only group was additionally compared with an ADHD-only group (Rubia et al. 2008, 2009a, b, 2010). Because four of the five cool EF studies were published by the same research group and included largely overlapping samples (Rubia et al. 2008, 2009a, b, 2010), we did not perform an ALE meta-analysis on cool EF fMRI studies in ODD/CD as at least five independent samples are required for this analysis (www.brainmap.org).

All studies investigated children/adolescents. For information regarding group characteristics of the included samples, see Tables 2 and 3. Reported differences in brain structures are bilateral, unless reported to be either left or right sided. Results are reported from WBA approaches, unless specified to be the result of ROI based analyses, see Tables 2 and 3.

Hot EF - ALE Meta-analysis Thirteen functional neuroimaging studies of hot EF were initially included in the meta- analysis. Due to the limited number of studies in ODD/CDonly (three studies) it was not possible to perform an ALE meta-analysis on studies comparing ODD/CD-only groups and controls, thus the ALE meta-analysis was performed on all ODD/CD samples, therewith including both individuals with and without comorbid ADHD.

There was a difference between the resulting maps of the ALE analysis with and without the ROI-only studies. Therefore, we reported the results from the map that included only WBA approach-based studies given the unbiased results. Total sample sizes for all included studies ranged between 30 and 75 individuals, adding up to a total of 423 subjects, of which 256 were patients and 167 were controls (age range 9-21 years). Three studies assessed a full male sample and six studies assessed a sample largely consisting of males (ranging from 56 to $79 \%$ ). The studies reported on different tasks associated with hot EF functions: three studies assessed processing of contingencies, two studies assessed passive viewing of emotional pictures, two studies assessed passive avoidance learning, one study assessed active viewing and rating of painful situations, and one study assessed implicit emotional processing.

The included studies provided a total of 68 foci of areas showing altered activity in individuals with ODD/CD with and without comorbid ADHD. The ALE analysis revealed five significant clusters representing areas with altered activity in patients with $\mathrm{ODD} / \mathrm{CD}$-only or ODD/CD+ADHD that were most consistently reported across the studies (see Fig. 3 for visualisation). The largest cluster $\left(416 \mathrm{~mm}^{3}\right)$ was located in the right globus pallidus, with two foci inside this cluster. The second $\left(328 \mathrm{~mm}^{3}\right)$ and third $\left(256 \mathrm{~mm}^{3}\right)$ clusters were located in the right and left amygdala, and contained three and two foci, respectively. The fourth cluster $\left(208 \mathrm{~mm}^{3}\right)$ was present in
Fig. 3 Results of the functional ALE meta-analysis of hot EF showing the clusters in right globus pallidus, bilateral amygdala, left caudate, and left fusiform gyrus $\left(p_{\text {corrected }}<0.05\right)$ superimposed on a structural scan in Talairach space. Top row: coronal view, bottom row: axial view. $L$ Left, $R$ Right

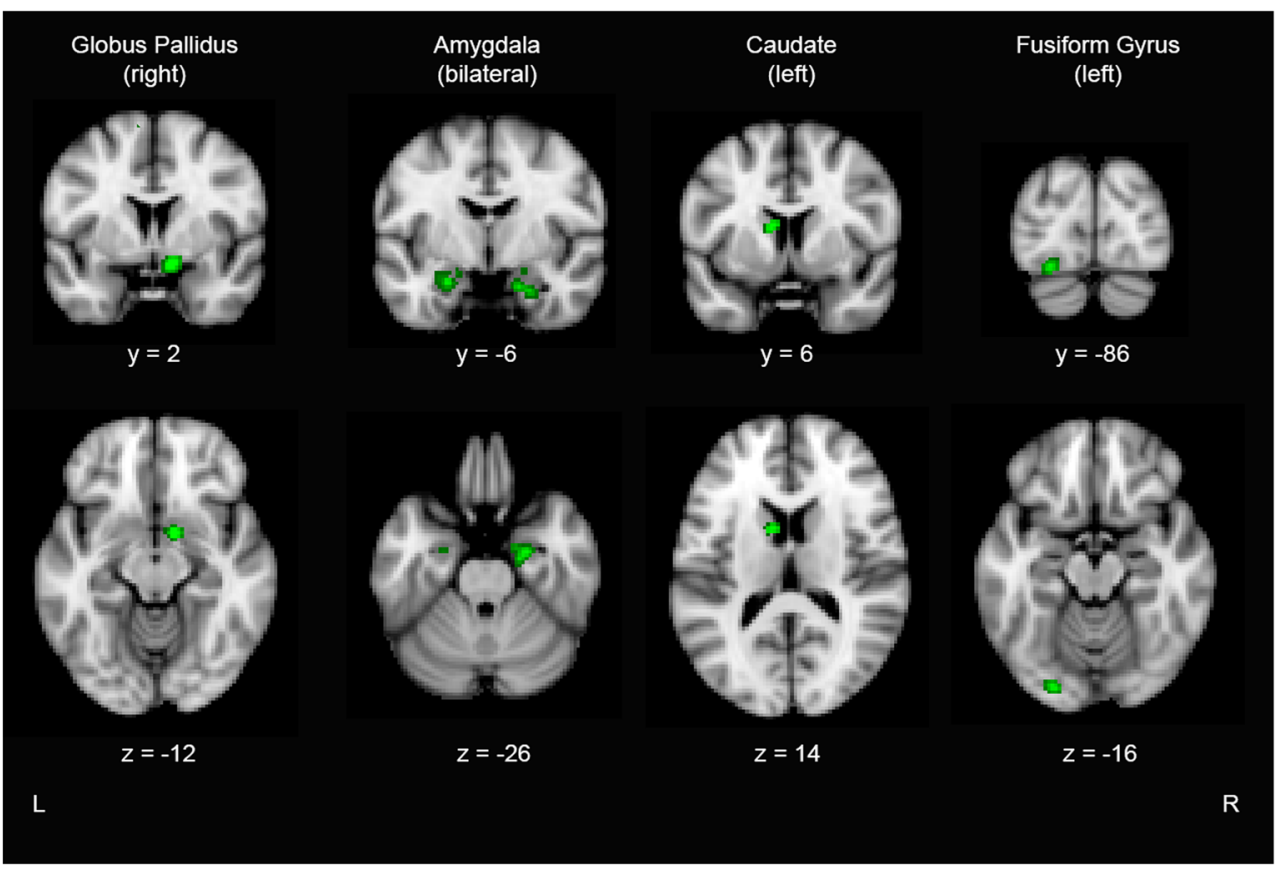


the left caudate, while the fifth cluster $\left(200 \mathrm{~mm}^{3}\right)$ was located in the left fusiform gyrus, both contained two foci.

Hot EF - Narrative Review Tables 2 and 3 provides an overview of investigated samples and approach (WBA or ROI) and summarizes the findings of the 13 included studies reporting on hot EF. Sample sizes ranged between 27 and 75 individuals, adding up to a total of 560 individuals, including 334 patients and 226 controls (age range 9-21 years). The samples consisted predominantly of males, with five studies using a full male sample, and the other eight studies using a sample largely consisting of males (ranging from 56 to $82 \%$ ). The studies reported on different tasks associated with hot EF functions: five studies assessed processing of contingencies, three studies assessed passive viewing of emotional pictures, two studies assessed passive avoidance learning, one study assessed active viewing and rating of painful situations, one study assessed implicit emotional processing, and one study assessed interference control with emotional stimuli. Of the 13 studies, 10 used a WBA approach and 3 only an ROI approach, resulting in different numbers of studies per investigated structure.

$O D D / C D$-only versus controls (3 studies). Two of the three studies reported abnormalities for ODD/CD-only compared to controls, of which one study reported lower activity in the right striatum, left amygdala, right insula, anterior cingulate cortex, and left medial and superior frontal gyrus, and the other study reported lower activity in the right lateral and medial orbitofrontal cortex.

$O D D / C D$-mixed and $O D D / C D+A D H D$ (10 studies). Studies assessing ODD/CD-mixed and ODD/CD+ADHD groups replicated findings of abnormal function of the striatum (six out of eight studies: three left, two right, one bilateral), including the caudate and putamen, and the amygdala (six out of nine studies: two left, two right, two bilateral). Additionally, functional abnormalities were reported in ODD/CD-mixed groups for the parietal cortex (three out of seven studies: two right, one left), which was not found for ODD/CD-only groups. This might indicate that the abnormal activity in the parietal cortex reflects the presence of comorbid ADHD rather than ODD/CD.

Correlational findings and specificity. Five studies investigated relations between activity of specific structures and $\mathrm{ODD} / \mathrm{CD}$ related symptoms. All five studies reported negative associations (thus a smaller volume related to more ODD/CD symptoms) between amygdala activity and ODD/CD related symptoms, one for an ODD/CD-only sample (left) and four for ODD/CD-mixed samples (two left, one right, one bilateral). Furthermore, two of the five studies reported negative associations between left and right anterior cingulate cortex activity and ODD/CD related symptoms, of which one assessed an ODD/CD-only sample and the other assessed an ODD/CD-mixed sample, respectively.
In terms of specificity, four studies investigated disorder specificity of the abnormalities compared to ADHD-only groups. Of these four studies, one study reported the lower activity in the orbitofrontal cortex in an ODD/CD-only group to be disorder specific, while the other study reported lower activity in the amygdala in an ODD/CD-mixed group to be disorder specific.

Hot EF - Summary Combining the results from the metaanalysis and the narrative review, hot EF functional neuroimaging studies most consistently implicated abnormalities of the amygdala and insula. Even though the meta-analysis implicated the left fusiform gyrus in ODD/CD, the narrative reviews did not show support for abnormalities in this area in $\mathrm{ODD} / \mathrm{CD}$.

Cool EF - Narrative Review Tables 2 and 3 provides an overview of investigated samples and approach (WBA or $\mathrm{ROI}$ ) and summarizes the findings of the five included studies reporting on cool EF. Sample sizes ranged between 21 and 53 individuals, with all studies assessing full male samples (age rang 9-17 years). The studies reported on different tasks associated with cool EF functions: one study assessed inhibitory control, one study assessed interference control, one study assessed attention allocation, one study assessed continuous performance, and one study assessed cognitive flexibility.

$O D D / C D$-only versus controls (5 studies). Four of the five studies reported less activation in the precuneus of ODD/CDonly groups, with two studies reporting the left precuneus, one the bilateral precuneus, and one the right precuneus. Furthermore, two of the five studies reported lower activity of the right insula, right anterior cingulate and right posterior cingulate cortex in ODD/CD-only.

Correlational findings and specificity. Two studies investigated relations between activity of specific structures and $\mathrm{ODD} / \mathrm{CD}$ related symptoms, of which one reported a negative association between dorsolateral prefrontal cortex and ODD/ $\mathrm{CD}$ related symptoms and the other reported no associations. In terms of specificity, four studies investigated disorder specificity of the abnormalities for ODD/CD as compared to ADHD-only. One of these four studies reported abnormal activity in the insula, anterior cingulate cortex, cerebellum and hippocampus to be specific for ODD/CD-only, while another study reported specificity for lower activity in the left parietaltemporal cluster and the right parietal lobe.

Cool EF - Summary The number of available studies into cool EF did not allow a meta-analysis to be conducted. Based on the narrative review, abnormalities in the precuneus were most consistently implicated in ODD/CD. 


\section{Integration of Quantitative and Qualitative Review Findings}

In order to determine which hot and cool EF related brain areas are implicated in ODD/CD, findings from both sMRI and fMRI meta-analyses as well as from the narrative reviews are integrated. Since the meta-analyses provide the strongest evidence for the convergence of findings, the main findings of the meta-analyses are discussed first. Second, findings from ODD/CD-only studies (10) are discussed, since these are not biased by comorbid ADHD, followed by findings from the ODD/CD-mixed and ODD/CD+ADHD studies (19). Finally, all these findings are integrated, resulting in an overall conclusion for neuroanatomical abnormalities related to ODD/ CD.

Table 4 provides an overview and integration of all reported abnormalities. For the overall conclusion, priority was given to results from the ALE meta-analyses, since these provide the most objective and strong evidence for abnormalities of a certain structure. Qualifications used in the overall conclusion encompassed 'strong evidence', 'some evidence', 'weak evidence' and 'no evidence'. For the qualification of 'strong evidence', the structure had to be reported in both of the ALE meta-analyses. Alternatively, the structure had to be reported in either one of the ALE meta-analyses, while being supported by at least half of the studies included in the narrative reviews (structural and functional) that investigated that structure. For the qualifications of 'some evidence' and 'weak evidence' at least a quarter of the studies included in the narrative review (structural and functional) that investigated that structure had to report abnormalities, while for 'some evidence' the reported abnormality needed additional support of one of the ALE meta-analyses. Finally, when less than a quarter of the studies that investigated that structure reported abnormalities, it was concluded that there was 'no evidence' for involvement of that structure in $\mathrm{ODD} / \mathrm{CD}$.

Meta-analyses Meta-analytic results implicated both structural and functional abnormalities for one of the hot EF related structures: the amygdala (left and bilateral, respectively). Other hot EF related areas were only implicated in the structural (insula, left frontal gyrus) or functional meta-analyses (striatum, left fusiform gyrus).

Narrative Review: ODD/CD-Only Versus Controls (10 Studies) Integration of the structural and functional findings implicated abnormalities in several hot EF related structures for $\mathrm{ODD} / \mathrm{CD}$, since these structures were reported in more than a quarter of the studies: the amygdala, right striatum, insula, and left frontal gyrus (see Table 4). For a range of structures there was only weak or no evidence for abnormalities in ODD/CD (see Table 4 for an overview).
Narrative Review: ODD/CD-Mixed and ODD/ CD+ADHD (19 Studies) Overall, findings from the ODD/ $\mathrm{CD}$-mixed and $\mathrm{ODD} / \mathrm{CD}+\mathrm{ADHD}$ groups again showed abnormalities in several hot EF related structures for ODD/CD: the amygdala, right striatum, insula and left frontal gyrus (see Table 4). For the amygdala and right striatum at least half of the structural and functional studies investigating those structures reported abnormalities, and for the insula and left frontal gyrus more than a quarter of the structural and functional studies investigating those structures reported abnormalities. Again, there was only weak or no evidence for involvement of a range of structures (see Table 4 for an overview).

Overall Conclusion and Specificity of Findings Integration of the structural and functional findings across the ODD/CDonly, ODD/CD-mixed and ODD/CD+ADHD samples provided strong evidence for abnormalities in structure and function of two hot EF related structures: the amygdala and the striatum (see Table 4). Additionally, there was some evidence for structural and functional abnormalities in two other hot EF related structures: the insula and the left frontal gyrus (see Table 4).

Further support for involvement of abnormalities of some of these structures in ODD/CD was established by comparing the results from studies using ODD/CD-only samples with the results from studies using ODD/CD-mixed and ODD/CD+ ADHD samples to see if results were mainly driven by $\mathrm{ODD} / \mathrm{CD}$, and reviewing the correlational and specificity findings. The involvement of the amygdala in ODD/CD was further supported by the finding that abnormalities were mainly driven by the studies including ODD/CD-only or ODD/ CD-mixed groups, and to a lesser extent by studies including ODD/CD+ADHD groups. Hence, it seems that abnormalities are related to $\mathrm{ODD} / \mathrm{CD}$ and not to comorbid ADHD. Furthermore, there was strong evidence from studies on specificity of abnormalities in the amygdala for ODD/CD as compared to ADHD-only, and there was strong evidence for an association between both amygdala structure and function and ODD/CD related symptoms. Similar evidence was reported for the insula, where again the results were mainly driven by studies including ODD/CD-only or ODD/CD-mixed groups, rather than by studies including ODD/CD+ADHD groups. Also, some evidence for an association between both insula structure and function and ODD/CD related symptoms was present. For the striatum there was some evidence for an association between abnormalities in this area and ODD/CD related symptoms. For the other areas (frontal and fusiform gyrus) there was no further supporting evidence from studies investigating specificity by comparing an ODD/CD-only sample with an ADHD-only sample, nor did correlational approaches provide evidence for associations between abnormalities in these areas and ODD/CD symptoms. 


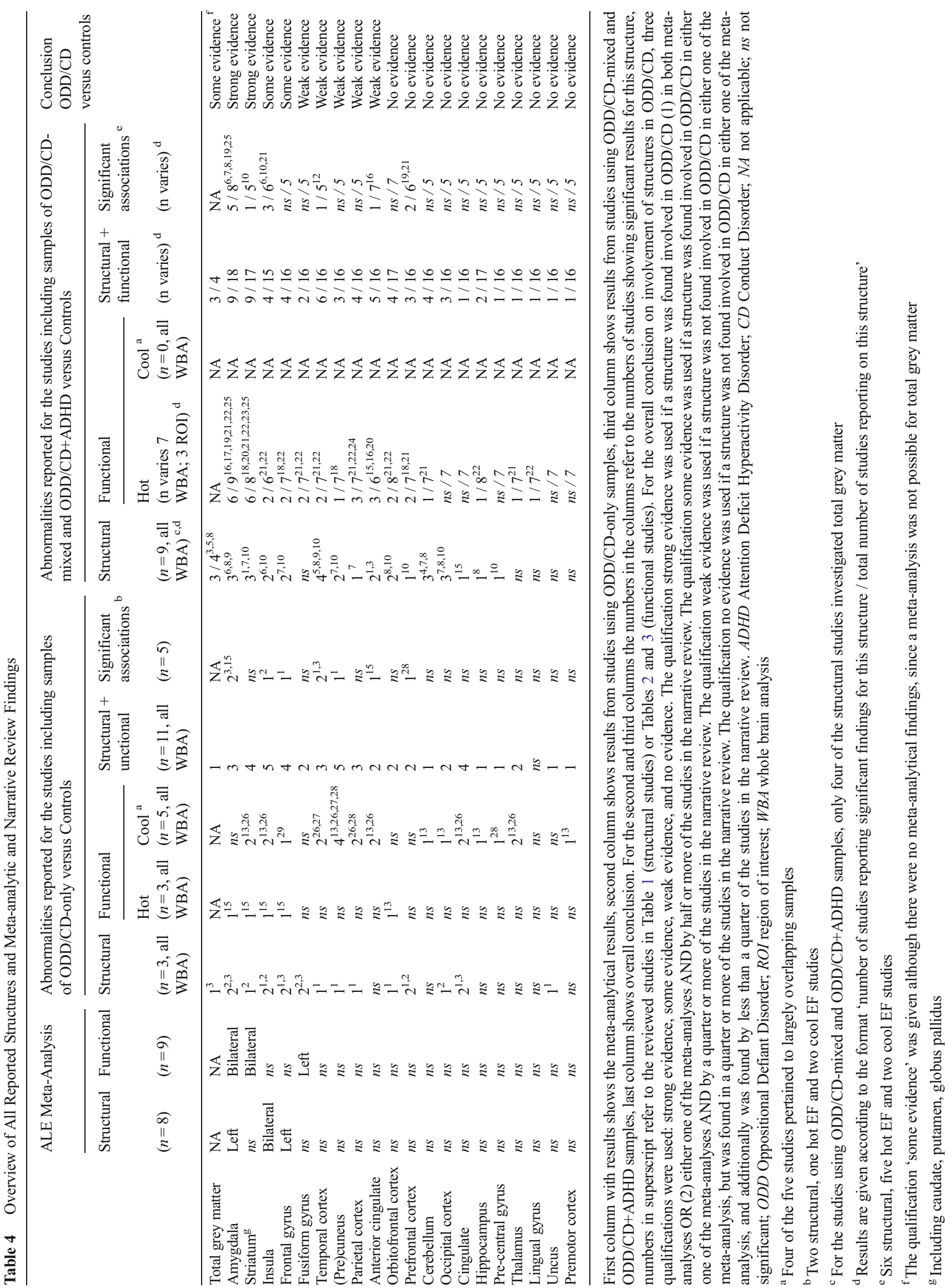


Finally, driven by results from the cool EF section using only ODD/CD-only samples, some evidence was found for abnormalities in a cool EF related structure in ODD/CD: the left precuneus. However, studies that compared ODD/CDonly samples with ADHD-only samples did not provide evidence for these abnormalities to be specific for ODD/CD, nor did studies that assessed associations provide evidence for precuneus volume to be related to ODD/CD symptoms. Taken together, our results show strong evidence for involvement of the amygdala and the striatum, some evidence for involvement of the insula and the frontal gyrus, and weak evidence for involvement of the precuneus in ODD/CD.

\section{Discussion}

We reviewed 29 structural and functional neuroimaging studies into hot and cool EF in ODD/CD (with and without ADHD) and performed two meta-analyses on subsets of these studies. We hypothesized that, compared to controls, individuals with ODD/CD (with and without ADHD) would show both structural and functional brain abnormalities in areas related to hot and cool EF. In addition, we investigated the specificity of these abnormalities by discussing results separately for studies including an ODD/CD-only group and results from studies including an ODD/CD-mixed or ODD/CD+ ADHD group, using a narrative approach. Furthermore, individuals with $\mathrm{ODD} / \mathrm{CD}$ were compared to individuals with ADHD. The results confirmed impairments in structure and function in most of the hypothesised hot EF related structures (i.e. amygdala, insula, and anterior cingulate), and to a lesser extent results confirmed such impairments in cool EF related structures (i.e. dorsolateral prefrontal cortex and its subcortical connections, including the cerebellum). Impairments were mainly present as reductions in volume or reductions in activity of the structures. Some of these abnormalities were reported to be specific to ODD/CD when compared to ADHD-only, or showed associations with ODD/CD symptom levels.

In terms of hot EF related neuroanatomical correlates, several structures were consistently reported to be associated with $\mathrm{ODD} / \mathrm{CD}$ (with and without ADHD) compared to controls (see Table 4 for an overview). This pattern of findings emerged irrespective of a structural or functional approach of the study. For two areas the combination of the narrative review and meta-analyses of both structural and functional MRI studies provided strong evidence of abnormalities in $\mathrm{ODD} / \mathrm{CD}$. These areas were the bilateral amygdala and the right striatum, including the caudate, putamen, and globus pallidus. In addition to these two areas, some evidence was provided for abnormalities of the bilateral insula in ODD/CD groups. For all three areas, the majority of studies reported reductions in volume and activity in $\mathrm{ODD} / \mathrm{CD}$ with and without ADHD groups compared to controls. Furthermore, for all three areas abnormalities appeared to be mainly driven by studies including ODD/CD-only or ODD/CD-mixed groups, and to a lesser extent by studies including ODD/CD+ADHD groups. Hence, it seems that ODD/CD may drive these abnormalities, rather than ADHD. For the amygdala, this claim is supported by strong evidence for the abnormalities to be specific for ODD/CD when compared to ADHD-only and by a significant association between abnormalities in the amygdala and ODD/CD symptoms in both ODD/CD-only and ODD/ CD-mixed groups. For the insula there was some evidence for specificity for ODD/CD compared to ADHD and some evidence for an association with ODD/CD symptoms. However, for the right striatum there was neither evidence for specificity for ODD/CD nor for an association between $\mathrm{ODD} / \mathrm{CD}$ related symptoms.

The finding of abnormalities in brain areas related to hot $\mathrm{EF}$ for $\mathrm{ODD} / \mathrm{CD}$ is in line with the abnormalities observed in $\mathrm{ODD} / \mathrm{CD}$ in terms of performance on hot EF related tasks. The amygdala, striatum, insula, and frontal gyrus are implicated in emotion processing (Schumann et al. 2011; Anderson and Kiehl 2012), reinforcement processing (Di Martino et al. 2008; Helie et al. 2013; Jessup and O'Doherty 2011), empathy (Gu et al. 2012, 2013; Lamm and Singer 2010), and introspection (Passamonti et al. 2012; Goldberg et al. 2006) in typically developing controls, respectively. There is robust support for abnormalities in emotion processing, altered reinforcement sensitivity, deficits in empathy and abnormalities in selfcontrol in individuals with ODD/CD (Blair 2013; Byrd et al. 2014; Burke et al. 2002).

In terms of cool EF related neuroanatomical correlates, few significant findings emerged. Partly, this was due to the limited number of functional neuroimaging studies into cool $\mathrm{EF}$ in individuals with $\mathrm{ODD} / \mathrm{CD}$. Almost all available functional studies were performed within the same sample, precluding a meta-analysis of these findings and restricting our approach to a narrative review of studies. For the left frontal gyrus some evidence for abnormalities in $\mathrm{ODD} / \mathrm{CD}$ was found. For the left precuneus weak evidence was found, however this structure was predominantly reported in studies using ODD/CD-only groups, suggesting involvement of this structure in ODD/CD. Future studies should confirm this claim. Surprisingly, there was no evidence for involvement of abnormalities of more typical cool EF structures, such as the dorsolateral prefrontal cortex or the cerebellum in ODD/CD. The lack of robust cerebellum-related results in $\mathrm{ODD} / \mathrm{CD}$ may be due to abnormalities in the cerebellum being more strongly related to comorbid ADHD, than to ODD/CD itself. Some support for this interpretation was found, since structural and functional abnormalities in the cerebellum were reported mainly in studies including ODD/CD+ADHD and ODD/CD-mixed groups, and not so much in studies including ODD/CD-only groups.

The absence of a significant relationship between brain areas related to cool $\mathrm{EF}$ and $\mathrm{ODD} / \mathrm{CD}$ is in line with the 
inconsistent findings on tasks assessing cool EF in this group. $\mathrm{ODD} / \mathrm{CD}$ seems to be associated with specific inhibitoryrelated abnormalities (Willoughby et al. 2011; Giedd et al. 2010; Hobson et al. 2011), rather than with more general cool EF abnormalities. This is emphasised by results from studies in other cool EF related domains, such as cognitive flexibility and working memory, that showed these domains to be intact in ODD/CD and to be associated with comorbid ADHD instead of ODD/CD (Dawel et al. 2012; Giedd et al. 2010; Hobson et al. 2011; Prencipe et al. 2011).

Although we have suggested that abnormalities in ODD/ $\mathrm{CD}$ are mainly associated with hot $\mathrm{EF}$, most of the reported hot EF brain areas are also involved in cool EF (see Fig. 4 for visualisation), implying an integrated model of abnormalities in both hot and cool EF, rather than a strict separation between these two. This is in line with models that propose abnormalities in both hot and cool EF circuits to underlie the behavioural characteristics of $\mathrm{ODD} / \mathrm{CD}$, such as the model by Blair (2005). The connectivity between the structures we found to be involved in ODD/CD (amygdala, insula, striatum, medial/ frontal gyrus, precuneus) has been extensively studied, and studies show that all these areas appear to be interconnected, albeit each to a different degree, and are found to subserve several different functions. For example, it is well established that the amygdala, as part of the limbic system, is critical for hot $\mathrm{EF}$, such as the processing of emotional information. Moreover, it also modulates behavioural responses subsequent to emotionally salient information, with the regulatory process being regarded as a cool EF related process (Schumann et al. 2011; Anderson and Kiehl 2012). The insula is also involved in emotional information processing, and is necessary for empathic pain perception and compassion, hence referring to hot EF, but also for the integration of

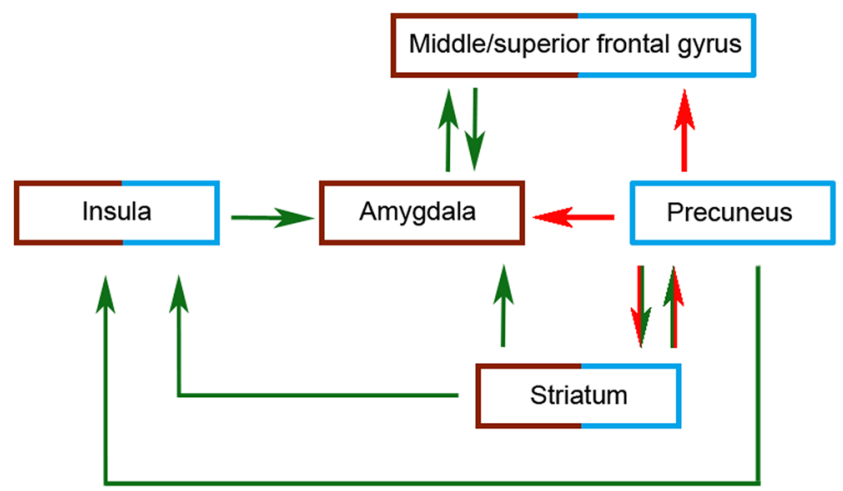

Fig. 4 Overview of the connectivity of areas involved in ODD/CD described in this review, based on connectivity studies in typically developing individuals (see main text). Green arrows indicate excitatory connectivity, red arrows indicate inhibitory connectivity, and arrows in both green and red indicate both excitatory and inhibitor connectivity. Red boxed areas exhibit mainly hot EF, blue boxed areas exhibit mainly cool $\mathrm{EF}$, and areas boxed in both red and blue exhibit a combination of hot and cool EF emotional and cognitive processes, thus referring to cool EF (Gu et al. 2012, 2013; Lamm and Singer 2010). The striatum, specifically the dorsal part (mainly caudate and putamen), is associated with inhibitory functions as well as with reinforcement learning, and thus referring to both cool and hot EF related processes, respectively (Di Martino et al. 2008; Helie et al. 2013; Jessup and O'Doherty 2011). The medial/superior frontal gyrus is associated with response inhibition and other cool EF functions, but also with introspection, which can be seen as a more hot EF related process (Passamonti et al. 2012; Goldberg et al. 2006). The precuneus, finally, is an area that is involved in reflective, self-related processing and awareness, incorporating both hot and cool EF processes (S. Zhang and $\mathrm{Li}$ 2012). Overall, it shows that our findings of abnormalities in the five abovementioned structures may lead to impairments that show striking parallels with the behavioural characteristics of individuals with ODD/CD, such as their difficulties in learning socially acceptable behaviour, and their tendency to contribute hostile intentions to peers in socially ambiguous situations (Byrd et al. 2014; Blair 2013; Burke et al. 2002). This supports an integrated model of hot and cool EF abnormalities in ODD/CD, for which the model by Blair (2005) is currently the most comprehensive and encompasses a full description of both circuits.

Our results regarding abnormalities in brain structure and function in ODD/CD are in line with models on brain functioning of other externalising disorders for which ODD and $\mathrm{CD}$ are risk-factors, such as antisocial personality disorder (ASPD) or psychopathy (American Psychiatric Association 2013). ASPD and psychopathy are characterised by symptoms of emotional detachment and impulsive behaviour, and the disregard for, or violation of, rights of others. The overlap in neurocognitive and behavioural abnormalities between ASPD, psychopathy and ODD/CD, suggests that these disorders may share an underlying aetiology. A recently proposed neurocognitive model of psychopathy includes the paralimbic system as a whole, comprising both the frontal lobe and the temporal lobe (including the amygdala). A review on psychopathy reported that most consistently implicated areas were the amygdala, anterior and posterior cingulate cortices, orbitofrontal cortex and adjacent (para)limbic structures (Yang and Raine 2009). Furthermore, these brain areas are also implicated in ASPD (Kiehl 2006; Blair 2010). Since we found some of these areas to be involved in $\mathrm{ODD} / \mathrm{CD}$, a shared aetiology with ASPD and psychopathy seems plausible.

\section{Research Agenda}

The goal of the current review was to aggregate and integrate findings from structural and functional studies on brain anatomical aspects of $\mathrm{ODD} / \mathrm{CD}$, to provide a complete overview of brain abnormalities associated with ODD/CD. During this process we encountered several issues in the field. Firstly, in 
our meta-analysis of hot EF functional studies, different results were obtained when the analyses were conducted with and without inclusion of ROI-based studies, suggesting that ROI-based studies may bias conclusions. We therefore restricted our hot EF meta-analysis to WBA approach-based studies, given their unbiased results. Future studies investigating brain abnormalities associated with ODD/CD should not only include ROI analyses, but would benefit from reporting (additional) whole brain analyses, rendering the results eligible to be included in a meta-analysis. In addition, because of the limited amount of studies into cool EF and the overlap in subjects in these studies, we were not able to perform a metaanalysis on these data; further research is needed in order to be able to draw firm conclusions on the possible involvement of cool EF abnormalities in ODD/CD, particularly since evidence from behavioural studies into cool EF impairments in $\mathrm{ODD} / \mathrm{CD}$ is inconclusive.

Secondly, in terms of sample characteristics, an important future direction is to study the role of callous-unemotional (CU) traits or psychopathic traits in individuals with ODD/ $\mathrm{CD}$. It has been reported that children with $\mathrm{CD}$ and high levels of CU traits show greater abnormalities in reward sensitivity, lower responsivity to treatment by parenting strategies, and are more resistant to psychosocial intervention than those with CD and few CU traits (Byrd et al. 2014). Another important issue in terms of the investigated samples is that most of the studies focussed on males, limiting generalization of current findings. This is emphasised by the differing pathways of brain development in boys and girls (Haney-Caron et al. 2014), and the fact that boys and girls seem to differ in the neural characteristics of ODD/CD (Hyatt et al. 2012). For example, when comparing male and female samples with $\mathrm{ODD} / \mathrm{CD}$-mixed, gender differences emerged for the insula (Fairchild et al. 2013a). Moreover, since substantial developmental changes take place during the transition from childhood to adulthood (Giedd et al. 2009), studies should take developmental patterns into account. This can be achieved by adhering to smaller age ranges when including participants, but also by matching on age or using large samples that allow developmental patterns to be studied. In the majority of the included studies, participant ages ranged between 10 and 17 years, which cover a substantial time of brain development. Furthermore, since ODD and CD are childhood disorders and are referred to as APD when persisting into adulthood, our findings may not be generalizable to older individuals.

Thirdly, at a statistical level, future studies should be more consistent in statistical corrections and thresholding. A significant proportion of the studies did not correct for multiple comparisons or Type 1 errors, nor applied cluster thresholding. Of these, most studies using a WBA approach did report Family Wise Error or False Discovery Rate corrected results, except for three structural and four functional studies (Bussing et al. 2002; Kruesi et al. 2004; Fairchild et al. 2013a; Marsh et al. 2013; Passamonti et al. 2010; White et al. 2013, 2014). However, most of these studies did set a minimum number of $>10$ contiguous voxels and lowered the statistical threshold to $p<.005$ (Marsh et al. 2013; White et al. 2013, 2014) or $p<.001$ (Passamonti et al. 2012; Fairchild et al. 2013a), leaving only two studies that reported at an uncorrected statistical threshold of $p<.05$. This implies that the results of both the meta-analyses and narrative reviews are in general reliable, but should be interpreted with some caution, since especially in WBA-based studies, these statistical methods are of importance (Chumbley and Friston 2009; Chumbley et al. 2010; Nichols and Hayasaka 2003).

Fourthly, future studies should look at other imaging techniques, such as DTI, to improve our knowledge on structural and functional brain abnormalities associated with ODD/CD. Up to date, eight studies have used DTI to study abnormalities in pathways in $\mathrm{ODD} / \mathrm{CD}$. Although there is relatively consistent evidence for involvement of the uncinate fasciculus (Wang et al. 2012; Finger et al. 2012; Haney-Caron et al. 2014; Passamonti et al. 2012; Sarkar et al. 2013; Zhang et al. 2014a), studies largely differed in terms of the white matter structures implicated in ODD/CD. Furthermore, while some studies report heightened DTI measures in ODD/CD (Passamonti et al. 2012; Sarkar et al. 2013; Zhang et al. 2014a, 2014b), other studies report lowered DTI measures in ODD/CD (Haney-Caron et al. 2014; Wang et al. 2012), or no differences at all (Finger et al. 2012). In addition to integrating findings of DTI studies, future studies could look at other imaging techniques, such as resting-state fMRI and combined sMRI-fMRI studies.

Finally, to advance our understanding of ODD and CD and the specificity of neural abnormalities future studies should assess well-defined and sufficiently sized samples. For example, the ideal study should at least compare ODD/CD-only, ADHD-only, comorbid ODD/CD+ADHD, and typically developing control groups with sufficient sample size, to investigate the possible confounding effect of comorbid ADHD. Furthermore, since there appears to be a difference in underlying mechanisms for childhood-onset and adolescent-limited ODD/CD (Moffitt 1993), future studies should differentiate between these types of ODD/CD. Moreover, studies should include separate groups of individuals with pure ODD and pure $\mathrm{CD}$, to investigate whether these disorders are indeed two ends of a spectrum or rather two separate disorders, since this is not completely evident from studies performed so far (Matthys et al. 2013; Rowe et al. 2010). A strict separation of the two disorders was not possible at the time of the current review, given that until the release of the DSM-5, a diagnosis of ODD was precluded when CD was present. Therefore most studies into CD did not investigate or report on ODD, possibly limiting the generalization of our findings to ODD-only and CD-only samples. In terms of ADHD comorbidity, most studies that reported on ODD/CD did not investigate (or report on) 
possible comorbidity with ADHD, hence, studies investigating $\mathrm{ODD} / \mathrm{CD}$-only samples are sparse. For this reason, we could not perform a meta-analysis on studies that compared groups with ODD/CD-only to groups with ADHD-only. Also, it would be of great interest to investigate whether ADHD comorbidity adds abnormalities associated with ADHD to those already present due to $\mathrm{ODD} / \mathrm{CD}$, or that $\mathrm{ODD} / \mathrm{CD}$ with comorbid ADHD is a genuinely separate disorder. Furthermore, in the current review we did not examine the impact of other common comorbidities such as anxiety or depression. This is especially important because these comorbidities are associated with abnormalities in some of the same brain areas that appear to be implicated in ODD/CD (Sterzer et al. 2005). Especially the amygdala is related to both anxiety and depressive disorder, albeit in an opposite direction, showing higher volumes, instead of the lower volumes, to be associated with ODD/CD (Machado-de-Sousa et al. 2014; Young et al. 2014). Furthermore, not all studies investigated (or reported on) (past) medication use, even though in almost all studies, MRI scanning was done in individuals free of medication at the time of testing (Rubia et al. 2013). Current and past mediation use should be incorporated in future studies, since this may have long-term effects on brain structure and function (Hart et al. 2013; Rubia et al. 2014).

\section{Conclusion}

We reviewed 29 studies on structural and functional neuroimaging in samples with ODD/CD with and without comorbid ADHD, and performed meta-analyses on a subset of eight structural studies and nine hot EF functional studies. Despite some limitations and heterogeneity amongst studies, results indicated that individuals with ODD/CD primarily show abnormalities in the bilateral amygdala, bilateral insula, right striatum and left medial/superior frontal gyrus as well as the left precuneus. Evidence of involvement of these areas was present in both structural and functional studies, and irrespective of whether the study included individuals with ODD/CDonly or with ODD/CD with comorbid ADHD. Our results show strong evidence of specificity for abnormalities in the amygdala for ODD/CD as compared to ADHD, and correlational studies further support the association between abnormalities in the amygdala and ODD/CD symptoms. Besides the left precuneus that was revealed by the narrative review of cool EF, there was no evidence for abnormalities in typical cool EF related structures, such as the cerebellum and dorsolateral prefrontal cortex. Our findings confirm the involvement of hot, and to a smaller extent cool EF associated brain areas in ODD/CD, and support an integrated model of both hot and cool deficits for ODD/CD (e.g. Blair 2005). The areas found associated with $\mathrm{ODD} / \mathrm{CD}$ are involved in emotion-processing, error monitoring, self-control, and empathic and social behaviour. It is precisely these functions that are impaired in children and adolescents with $\mathrm{ODD} / \mathrm{CD}$, and that result in difficulties learning socially accepted behaviours and reactions, attributing hostile intentions to others, and preferring aggressive solutions to social dilemmas.

Open Access This article is distributed under the terms of the Creative Commons Attribution 4.0 International License (http:// creativecommons.org/licenses/by/4.0/), which permits unrestricted use, distribution, and reproduction in any medium, provided you give appropriate credit to the original author(s) and the source, provide a link to the Creative Commons license, and indicate if changes were made.

\section{References}

American Psychiatric Association (2013) Diagnostic and statistical manual of mental disorders: DSM-5, 5th edn. Arlington: American Psychiatric Publishing, Inc.

Anderson, N. E., \& Kiehl, K. A. (2012). The psychopath magnetized: insights from brain imaging. Trends in Cognitive Sciences, 16(1), 52-60. doi:10.1016/j.tics.2011.11.008.

Anderson, N. E., \& Kiehl, K. A. (2013). Psychopathy: developmental perspectives and their implications for treatment. Restorative Neurology and Neuroscience. doi:10.3233/rnn-139001.

Anderson, V. A., Jacobs, R., \& Anderson, P. J. (2008). Executive functions and frontal lobes: A lifespan perspective. Hove: Psychology Press.

Blair, R. J. (2005). Applying a cognitive neuroscience perspective to the disorder of psychopathy. Development and Psychopathology, 17(3), 865-891. doi:10.1017/s0954579405050418.

Blair, R. J. (2010). Neuroimaging of psychopathy and antisocial behavior: a targeted review. Current Psychiatry Reports, 12(1), 76-82. doi:10.1007/s11920-009-0086-x.

Blair, R. J. (2013). The neurobiology of psychopathic traits in youths. Nature Reviews Neuroscience, 14(11), 786-799. doi:10.1038/ nrn3577.

Blair, R. J., \& Lee, T. M. (2013). The social cognitive neuroscience of aggression, violence, and psychopathy. Social Neuroscience, 8(2), 108-111. doi:10.1080/17470919.2012.757869.

Boylan, K., Vaillancourt, T., Boyle, M., \& Szatmari, P. (2007). Comorbidity of internalizing disorders in children with oppositional defiant disorder. European Child and Adolescent Psychiatry, 16(8), 484-494. doi:10.1007/s00787-007-0624-1.

Burke, J. D., Loeber, R., \& Birmaher, B. (2002). Oppositional defiant disorder and conduct disorder: a review of the past 10 years, part II. Journal of the American Academy of Child and Adolescent Psychiatry, 41(11), 1275-1293. doi:10.1097/00004583200211000-00009.

Bussing, R., Grudnik, J., Mason, D., Wasiak, M., \& Leonard, C. (2002). ADHD and conduct disorder: an MRI study in a community sample. The World Journal of Biological Psychiatry, 3(4), 216-220.

Byrd, A. L., Loeber, R., \& Pardini, D. A. (2014). Antisocial behavior, psychopathic features and abnormalities in reward and punishment processing in youth. Clinical Child and Family Psychology Review, 17(2), 125-156. doi:10.1007/s10567-013-0159-6.

Chumbley, J. R., \& Friston, K. J. (2009). False discovery rate revisited: FDR and topological inference using Gaussian random fields. NeuroImage, 44(1), 62-70. doi:10.1016/j.neuroimage.2008.05.021.

Chumbley, J. R., Worsley, K., Flandin, G., \& Friston, K. (2010). Topological FDR for neuroimaging. NeuroImage, 49(4), 30573064. doi:10.1016/j.neuroimage.2009.10.090. 
Cohn, M. D., Veltman, D. J., Pape, L. E., van Lith, K., Vermeiren, R. R., van den Brink, W., et al. (2014). Incentive processing in persistent disruptive behavior and psychopathic traits: a functional magnetic resonance imaging study in adolescents. Biological Psychiatry. doi: 10.1016/j.biopsych.2014.08.017.

Connor, D. F., Steeber, J., \& McBurnett, K. (2010). A review of attentiondeficit/hyperactivity disorder complicated by symptoms of oppositional defiant disorder or conduct disorder. Journal of Developmental and Behavioral Pediatrics, 31(5), 427-440. doi:10. 1097/DBP.0b013e3181e121bd.

Coolidge, F. L., Thede, L. L., \& Young, S. E. (2000). Heritability and the comorbidity of attention deficit hyperactivity disorder with behavioral disorders and executive function deficits: a preliminary investigation. Developmental Neuropsychology, 17(3), 273-287. doi:10. 1207/s15326942dn1703 1.

Crowe, S. L., \& Blair, R. J. (2008). The development of antisocial behavior: what can we learn from functional neuroimaging studies? Development and Psychopathology, 20(4), 1145-1159. doi:10. 1017/s0954579408000540.

Dawel, A., O’Kearney, R., McKone, E., \& Palermo, R. (2012). Not just fear and sadness: meta-analytic evidence of pervasive emotion recognition deficits for facial and vocal expressions in psychopathy. Neuroscience and Biobehavioral Reviews, 36(10), 2288-2304. doi:10.1016/j.neubiorev.2012.08.006.

Di Martino, A., Scheres, A., Margulies, D. S., Kelly, A. M., Uddin, L. Q., Shehzad, Z., et al. (2008). Functional connectivity of human striatum: a resting state FMRI study. Cerebral Cortex, 18(12), 2735 2747. doi:10.1093/cercor/bhn041.

Diamond, A. (2013). Executive functions. Annual Review of Psychology, 64, 135-168. doi:10.1146/annurev-psych-113011-143750.

Dolan, M., \& Lennox, C. (2013). Cool and hot executive function in conduct-disordered adolescents with and without co-morbid attention deficit hyperactivity disorder: relationships with externalizing behaviours. Psychological Medicine, 1-10. doi:10.1017/ s0033291712003078.

Eickhoff, S. B., Laird, A. R., Grefkes, C., Wang, L. E., Zilles, K., \& Fox, P. T. (2009). Coordinate-based activation likelihood estimation meta-analysis of neuroimaging data: a random-effects approach based on empirical estimates of spatial uncertainty. Human Brain Mapping, 30(9), 2907-2926. doi:10.1002/hbm.20718.

Eickhoff, S. B., Bzdok, D., Laird, A. R., Kurth, F., \& Fox, P. T. (2012). Activation likelihood estimation meta-analysis revisited. NeuroImage, 59(3), 2349-2361. doi:10.1016/j.neuroimage.2011. 09.017.

Fahim, C., He, Y., Yoon, U., Chen, J., Evans, A., \& Perusse, D. (2011). Neuroanatomy of childhood disruptive behavior disorders. Aggressive Behavior, 37(4), 326-337. doi:10.1002/ab.20396.

Fairchild, G., Passamonti, L., Hurford, G., Hagan, C. C., von dem Hagen, E. A., van Goozen, S. H., et al. (2011). Brain structure abnormalities in early-onset and adolescent-onset conduct disorder. The American Journal of Psychiatry, 168(6), 624-633. doi:10.1176/appi.ajp.2010. 10081184.

Fairchild, G., Hagan, C. C., Walsh, N. D., Passamonti, L., Calder, A. J., \& Goodyer, I. M. (2013a). Brain structure abnormalities in adolescent girls with conduct disorder. Journal of Child Psychology and Psychiatry, 54(1), 86-95. doi:10.1111/j.1469-7610.2012.02617.x.

Fairchild, G., van Goozen, S. H., Calder, A. J., \& Goodyer, I. M. (2013b). Research review: evaluating and reformulating the developmental taxonomic theory of antisocial behaviour. Journal of Child Psychology and Psychiatry, 54(9), 924-940. doi:10.1111/jcpp. 12102.

Finger, E. C., Marsh, A. A., Mitchell, D. G., Reid, M. E., Sims, C., Budhani, S., et al. (2008). Abnormal ventromedial prefrontal cortex function in children with psychopathic traits during reversal learning. Archives of General Psychiatry, 65(5), 586-594.
Finger, E. C., Marsh, A. A., Blair, K. S., Reid, M. E., Sims, C., Ng, P., et al. (2011). Disrupted reinforcement signaling in the orbitofrontal cortex and caudate in youths with conduct disorder or oppositional defiant disorder and a high level of psychopathic traits. The American Journal of Psychiatry, 168(2), 152-162. doi:10.1176/ appi.ajp.2010.10010129.

Finger, E. C., Marsh, A., Blair, K. S., Majestic, C., Evangelou, I., Gupta, K., et al. (2012). Impaired functional but preserved structural connectivity in limbic white matter tracts in youth with conduct disorder or oppositional defiant disorder plus psychopathic traits. Psychiatry Research, 202(3), 239-244. doi:10.1016/j.pscychresns.2011.11. 002.

Fox, P. T., Parsons, L. M., \& Lancaster, J. L. (1998). Beyond the single study: function/location metanalysis in cognitive neuroimaging. Current Opinion in Neurobiology, 8(2), 178-187.

Gatzke-Kopp, L. M., Beauchaine, T. P., Shannon, K. E., Chipman, J., Fleming, A. P., Crowell, S. E., et al. (2009). Neurological correlates of reward responding in adolescents with and without externalizing behavior disorders. Journal of Abnormal Psychology, 118(1), 203213. doi:10.1037/a0014378.

Giedd, J. N., Lalonde, F. M., Celano, M. J., White, S. L., Wallace, G. L., Lee, N. R., et al. (2009). Anatomical brain magnetic resonance imaging of typically developing children and adolescents. Journal of the American Academy of Child and Adolescent Psychiatry, 48(5), 465-470. doi:10.1097/CHI.0b013e31819f2715.

Giedd, J. N., Stockman, M., Weddle, C., Liverpool, M., AlexanderBloch, A., Wallace, G. L., et al. (2010). Anatomic magnetic resonance imaging of the developing child and adolescent brain and effects of genetic variation. Neuropsychology Review, 20(4), 349361. doi:10.1007/s11065-010-9151-9.

Goldberg, I. I., Harel, M., \& Malach, R. (2006). When the brain loses its self: prefrontal inactivation during sensorimotor processing. Neuron, 50(2), 329-339. doi:10.1016/j.neuron.2006.03.015.

Gu, X., Gao, Z., Wang, X., Liu, X., Knight, R. T., Hof, P. R., et al. (2012). Anterior insular cortex is necessary for empathetic pain perception. Brain, 135(Pt 9), 2726-2735. doi:10.1093/brain/aws199.

Gu, X., Hof, P. R., Friston, K. J., \& Fan, J. (2013). Anterior insular cortex and emotional awareness. The Journal of Comparative Neurology. doi:10.1002/cne. 23368 .

Hamilton, S. S., \& Armando, J. (2008). Oppositional defiant disorder. American Family Physician, 78(7), 861-866.

Haney-Caron, E., Caprihan, A., \& Stevens, M. C. (2014). DTI-measured white matter abnormalities in adolescents with Conduct Disorder. Journal of Psychiatric Research, 48(1), 111-120. doi:10.1016/j. jpsychires.2013.09.015.

Hart, H., Radua, J., Nakao, T., Mataix-Cols, D., \& Rubia, K. (2013). Meta-analysis of functional magnetic resonance imaging studies of inhibition and attention in attention-deficit/hyperactivity disorder: exploring task-specific, stimulant medication, and age effects. JAMA Psychiatry, 70(2), 185-198. doi:10.1001/jamapsychiatry. 2013.277.

Helie, S., Chakravarthy, S., \& Moustafa, A. A. (2013). Exploring the cognitive and motor functions of the basal ganglia: an integrative review of computational cognitive neuroscience models. Frontiers in Computational Neuroscience, 7, 174. doi:10.3389/fncom.2013. 00174.

Herpertz, S. C., Huebner, T., Marx, I., Vloet, T. D., Fink, G. R., Stoecker, T., et al. (2008). Emotional processing in male adolescents with childhood-onset conduct disorder. Journal of Child Psychology and Psychiatry, 49(7), 781-791. doi:10.1111/j.1469-7610.2008. 01905.x.

Hobson, C. W., Scott, S., \& Rubia, K. (2011). Investigation of cool and hot executive function in ODD/CD independently of ADHD. Journal of Child Psychology and Psychiatry, 52(10), 1035-1043. doi:10.1111/j.1469-7610.2011.02454.x. 
Huebner, T., Vloet, T. D., Marx, I., Konrad, K., Fink, G. R., Herpertz, S. C., et al. (2008). Morphometric brain abnormalities in boys with conduct disorder. Journal of the American Academy of Child and Adolescent Psychiatry, 47(5), 540-547. doi:10.1097/CHI. 0b013e3181676545.

Hummer, T. A., Wang, Y., Kronenberger, W. G., Dunn, D. W., \& Mathews, V. P. (2015). The relationship of brain structure to age and executive functioning in adolescent disruptive behavior disorder. Psychiatry Research, 231(3), 210-217. doi:10.1016/j. pscychresns.2014.11.009.

Hyatt, C. J., Haney-Caron, E., \& Stevens, M. C. (2012). Cortical thickness and folding deficits in conduct-disordered adolescents. Biological Psychiatry, 72(3), 207-214. doi:10.1016/j.biopsych. 2011.11.017.

Jessup, R. K., \& O'Doherty, J. P. (2011). Human dorsal striatal activity during choice discriminates reinforcement learning behavior from the gambler's fallacy. The Journal of Neuroscience, 31(17), 62966304. doi:10.1523/jneurosci.6421-10.2011.

Kalnin, A. J., Edwards, C. R., Wang, Y., Kronenberger, W. G., Hummer, T. A., Mosier, K. M., et al. (2011). The interacting role of media violence exposure and aggressive-disruptive behavior in adolescent brain activation during an emotional Stroop task. Psychiatry Research, 192(1), 12-19. doi:10.1016/j.pscychresns.2010.11.005.

Kerr, A., \& Zelazo, P. D. (2004). Development of "hot" executive function: the children's gambling task. Brain and Cognition, 55(1), 148157. doi:10.1016/s0278-2626(03)00275-6.

Kiehl, K. A. (2006). A cognitive neuroscience perspective on psychopathy: evidence for paralimbic system dysfunction. Psychiatry Research, 142(2-3), 107-128. doi:10.1016/j.psychres.2005.09.013.

Kimonis, E. R., \& Frick, P. J. (2010). Oppositional defiant disorder and conduct disorder grown-up. Journal of Developmental and Behavioral Pediatrics, 31(3), 244-254. doi:10.1097/DBP. 0b013e3181d3d320.

Kruesi, M. J., Casanova, M. F., Mannheim, G., \& Johnson-Bilder, A. (2004). Reduced temporal lobe volume in early onset conduct disorder. Psychiatry Research, 132(1), 1-11. doi:10.1016/j. pscychresns.2004.07.002.

Lahey, B. B., \& Waldman, I. D. (2012). Annual research review: phenotypic and causal structure of conduct disorder in the broader context of prevalent forms of psychopathology. Journal of Child Psychology and Psychiatry, 53(5), 536-557. doi:10.1111/j.1469-7610.2011. 02509.x.

Laird, A. R., Fox, P. M., Price, C. J., Glahn, D. C., Uecker, A. M., Lancaster, J. L., et al. (2005). ALE meta-analysis: controlling the false discovery rate and performing statistical contrasts. Human Brain Mapping, 25(1), 155-164. doi:10.1002/hbm.20136.

Laird, A. R., Robinson, J. L., McMillan, K. M., Tordesillas-Gutierrez, D., Moran, S. T., Gonzales, S. M., et al. (2010). Comparison of the disparity between Talairach and MNI coordinates in functional neuroimaging data: validation of the Lancaster transform. NeuroImage, 51(2), 677-683. doi:10.1016/j.neuroimage.2010.02.048.

Lamm, C., \& Singer, T. (2010). The role of anterior insular cortex in social emotions. Brain Structure and Function, 214(5-6), 579591. doi:10.1007/s00429-010-0251-3.

Lancaster, J. L., Tordesillas-Gutierrez, D., Martinez, M., Salinas, F., Evans, A., Zilles, K., et al. (2007). Bias between MNI and Talairach coordinates analyzed using the ICBM-152 brain template. Human Brain Mapping, 28(11), 1194-1205. doi:10.1002/hbm. 20345.

Loeber, R., Burke, J. D., Lahey, B. B., Winters, A., \& Zera, M. (2000). Oppositional defiant and conduct disorder: a review of the past 10 years, part I. Journal of the American Academy of Child and Adolescent Psychiatry, 39(12), 1468-1484. doi:10.1097/ 00004583-200012000-00007.

Loeber, R., Slot, N. W., Van der Laan, P., \& Hoeve, M. (2008). Tomorrow's criminals. Farnham: Ashgate Publishing Limited.
Loeber, R., Burke, J., \& Pardini, D. A. (2009). Perspectives on oppositional defiant disorder, conduct disorder, and psychopathic features. Journal of Child Psychology and Psychiatry, 50(1-2), 133-142. doi: 10.1111/j.1469-7610.2008.02011.x. Research Support, N.I.H., Extramural Review.

Machado-de-Sousa, J. P., Osorio Fde, L., Jackowski, A. P., Bressan, R. A., Chagas, M. H., Torro-Alves, N., et al. (2014). Increased amygdalar and hippocampal volumes in young adults with social anxiety. PLoS One, 9(2), e88523. doi:10.1371/journal.pone. 0088523.

Marsh, A. A., Finger, E. C., Mitchell, D. G., Reid, M. E., Sims, C., Kosson, D. S., et al. (2008). Reduced amygdala response to fearful expressions in children and adolescents with callous-unemotional traits and disruptive behavior disorders. The American Journal of Psychiatry, 165(6), 712-720. doi:10.1176/appi.ajp.2007.07071145.

Marsh, A. A., Finger, E. C., Fowler, K. A., Adalio, C. J., Jurkowitz, I. T. N., Schechter, J. C., et al. (2013). Empathic responsiveness in amygdala and anterior cingulate cortex in youths with psychopathic traits. Journal of Child Psychology and Psychiatry, 54(8), 900-910. doi: 10.1111/jcpp.12063.

Matthys, W., Vanderschuren, L. J., \& Schutter, D. J. (2013). The neurobiology of oppositional defiant disorder and conduct disorder: altered functioning in three mental domains. Development and Psychopathology, 25(1), 193-207. doi:10.1017/ s0954579412000272.

McAlonan, G. M., Cheung, V., Cheung, C., Chua, S. E., Murphy, D. G., Suckling, J., et al. (2007). Mapping brain structure in attention deficit-hyperactivity disorder: a voxel-based MRI study of regional grey and white matter volume. Psychiatry Research, 154(2), 171180. doi:10.1016/j.pscychresns.2006.09.006.

Michalska, K. J., Decety, J., Zeffiro, T. A., \& Lahey, B. B. (2015). Association of regional gray matter volumes in the brain with disruptive behavior disorders in male and female children. NeuroImage, 7, 252-257. doi:10.1016/j.nicl.2014.12.012.

Moffitt, T. E. (1993). Adolescence-limited and life-course-persistent antisocial behavior: a developmental taxonomy. Psychological Review, 100(4), 674-701.

Newman, J. P., Kosson, D. S. (1986). Passive avoidance learning in psychopathic and nonpsychopathic offenders. Journal of Abnormal Psychology, 95, 252-256

Nichols, T., \& Hayasaka, S. (2003). Controlling the familywise error rate in functional neuroimaging: a comparative review. Statistical Methods in Medical Research, 12(5), 419-446.

Oosterlaan, J., Logan, G. D., \& Sergeant, J. A. (1998). Response inhibition in $\mathrm{AD} / \mathrm{HD}, \mathrm{CD}$, comorbid $\mathrm{AD} / \mathrm{HD}+\mathrm{CD}$, anxious, and control children: a meta-analysis of studies with the stop task. Journal of Child Psychology and Psychiatry, 39(3), 411-425.

Passamonti, L., Fairchild, G., Goodyer, I. M., Hurford, G., Hagan, C. C., Rowe, J. B., et al. (2010). Neural abnormalities in early-onset and adolescence-onset conduct disorder. Archives of General Psychiatry, 67(7), 729-738. doi:10.1001/archgenpsychiatry.2010.75.

Passamonti, L., Fairchild, G., Fornito, A., Goodyer, I. M., Nimmo-Smith, I., Hagan, C. C., et al. (2012). Abnormal anatomical connectivity between the amygdala and orbitofrontal cortex in conduct disorder. PLoS One, 7(11), e48789. doi:10.1371/journal.pone.0048789.

Pennington, B. F., \& Ozonoff, S. (1996). Executive functions and developmental psychopathology. Journal of Child Psychology and Psychiatry, 37(1), 51-87.

Prencipe, A., Kesek, A., Cohen, J., Lamm, C., Lewis, M. D., \& Zelazo, P. D. (2011). Development of hot and cool executive function during the transition to adolescence. Journal of Experimental Child Psychology, 108(3), 621-637. doi:10.1016/j.jecp.2010.09.008.

Quay, H. C. (1965). Psychopathic Personality as Pathological Stimulation-seeking. The American Journal of Psychiatry, 122 180-183 
Quay, H. C. (1993). The psychobiology of undersocialized aggressive conduct disorder: A theoretical perspective. Development and Psychopathology, 5(1-2), 165-180

Quay, H. C., \& Hogan, A. E. (1999). Handbook of disruptive behaviour disorders. US: Springer.

Rowe, R., Maughan, B., Pickles, A., Costello, E. J., \& Angold, A. (2002). The relationship between DSM-IV oppositional defiant disorder and conduct disorder: findings from the Great Smoky Mountains Study. Journal of Child Psychology and Psychiatry, 43(3), 365-373.

Rowe, R., Costello, E. J., Angold, A., Copeland, W. E., \& Maughan, B. (2010). Developmental pathways in oppositional defiant disorder and conduct disorder. Journal of Abnormal Psychology, 119(4), 726-738. doi:10.1037/a0020798.

Rubia, K. (2011). "Cool" inferior frontostriatal dysfunction in attentiondeficit/hyperactivity disorder versus "hot" ventromedial orbitofrontal-limbic dysfunction in conduct disorder: a review. Biological Psychiatry, 69(12), e69-e87. doi:10.1016/j.biopsych. 2010.09.023.

Rubia, K., Halari, R., Smith, A. B., Mohammed, M., Scott, S., Giampietro, V., et al. (2008). Dissociated functional brain abnormalities of inhibition in boys with pure conduct disorder and in boys with pure attention deficit hyperactivity disorder. The American Journal of Psychiatry, 165(7), 889-897. doi:10.1176/appi.ajp. 2008.07071084.

Rubia, K., Halari, R., Smith, A. B., Mohammad, M., Scott, S., \& Brammer, M. J. (2009a). Shared and disorder-specific prefrontal abnormalities in boys with pure attention-deficit/hyperactivity disorder compared to boys with pure CD during interference inhibition and attention allocation. Journal of Child Psychology and Psychiatry, 50(6), 669-678. doi:10.1111/j.1469-7610.2008.02022. $\mathrm{x}$.

Rubia, K., Smith, A. B., Halari, R., Matsukura, F., Mohammad, M., Taylor, E., et al. (2009b). Disorder-specific dissociation of orbitofrontal dysfunction in boys with pure conduct disorder during reward and ventrolateral prefrontal dysfunction in boys with pure ADHD during sustained attention. The American Journal of Psychiatry, 166(1), 83-94. doi:10.1176/appi.ajp.2008.08020212.

Rubia, K., Halari, R., Cubillo, A., Mohammad, A. M., Scott, S., \& Brammer, M. (2010). Disorder-specific inferior prefrontal hypofunction in boys with pure attention-deficit/hyperactivity disorder compared to boys with pure conduct disorder during cognitive flexibility. Human Brain Mapping, 31(12), 1823-1833. doi:10. 1002/hbm.20975.

Rubia, K., Alegria, A. A., Cubillo, A. I., Smith, A. B., Brammer, M. J., \& Radua, J. (2013). Effects of stimulants on brain function in attentiondeficit/hyperactivity disorder: a systematic review and meta-analysis. Biological Psychiatry. doi:10.1016/j.biopsych.2013.10.016.

Rubia, K., Alegria, A., \& Brinson, H. (2014). Imaging the ADHD brain: disorder-specificity, medication effects and clinical translation. Expert Review of Neurotherapeutics, 14(5), 519-538. doi:10.1586/ 14737175.2014.907526.

Sarkar, S., Craig, M. C., Catani, M., Dell'Acqua, F., Fahy, T., Deeley, Q., et al. (2013). Frontotemporal white-matter microstructural abnormalities in adolescents with conduct disorder: a diffusion tensor imaging study. Psychological Medicine, 43(2), 401-411. doi:10. 1017/s003329171200116x.

Sasayama, D., Hayashida, A., Yamasue, H., Harada, Y., Kaneko, T., Kasai, K., et al. (2010). Neuroanatomical correlates of attentiondeficit-hyperactivity disorder accounting for comorbid oppositional defiant disorder and conduct disorder. Psychiatry and Clinical Neurosciences, 64(4), 394-402. doi:10.1111/j.1440-1819.2010. 02102.x.

Schumann, C. M., Bauman, M. D., \& Amaral, D. G. (2011). Abnormal structure or function of the amygdala is a common component of neurodevelopmental disorders. Neuropsychologia, 49(4), 745-759. doi:10.1016/j.neuropsychologia.2010.09.028.
Sterzer, P., \& Stadler, C. (2009). Neuroimaging of aggressive and violent behaviour in children and adolescents. [Review]. Frontiers in Behavioral Neuroscience, 3, doi:10.3389/neuro.08.035.2009.

Sterzer, P., Stadler, C., Krebs, A., Kleinschmidt, A., \& Poustka, F. (2005). Abnormal neural responses to emotional visual stimuli in adolescents with conduct disorder. Biological Psychiatry, 57(1), 7-15. doi: 10.1016/j.biopsych.2004.10.008.

Sterzer, P., Stadler, C., Poustka, F., \& Kleinschmidt, A. (2007). A structural neural deficit in adolescents with conduct disorder and its association with lack of empathy. NeuroImage, 37(1), 335-342. doi: 10.1016/j.neuroimage.2007.04.043.

Stevens, M. C., \& Haney-Caron, E. (2012). Comparison of brain volume abnormalities between ADHD and conduct disorder in adolescence. Journal of Psychiatry and Neuroscience, 37(6), 389-398.

Turkeltaub, P. E., Eden, G. F., Jones, K. M., \& Zeffiro, T. A. (2002). Meta-analysis of the functional neuroanatomy of single-word reading: method and validation. NeuroImage, $16(3 \mathrm{Pt} 1), 765-780$.

Turkeltaub, P. E., Eickhoff, S. B., Laird, A. R., Fox, M., Wiener, M., \& Fox, P. (2012). Minimizing within-experiment and within-group effects in Activation Likelihood Estimation meta-analyses. Human Brain Mapping, 33(1), 1-13. doi:10.1002/hbm.21186.

Van Goozen, S. H. M., Cohen-Kettenis, P. T., Snoek, H., Matthys, W., Swaab-Barneveld, H., \& Van Engeland, H., et al. (2004). Executive functioning in children: a comparison of hospitalised ODD and ODD/ADHD children and normal controls. Journal of Child Psychology and Psychiatry, 45(2), 284-292 doi:10.1111/j.14697610.2004.00220.x.

Wang, Y., Horst, K. K., Kronenberger, W. G., Hummer, T. A., Mosier, K. M., Kalnin, A. J., et al. (2012). White matter abnormalities associated with disruptive behavior disorder in adolescents with and without attention-deficit/hyperactivity disorder. Psychiatry Research: Neuroimaging, 202(3), 245-251. Article.

Waschbusch, D. A. (2002). A meta-analytic examination of comorbid hyperactive-impulsive-attention problems and conduct problems. Psychological Bulletin, 128(1), 118-150. doi:10.1037/0033-2909. 128.1.118.

White, S. F., Pope, K., Sinclair, S., Fowler, K. A., Brislin, S. J., Williams, W. C., et al. (2013). Disrupted expected value and prediction error signaling in youths with disruptive behavior disorders during a passive avoidance task. The American Journal of Psychiatry, 170(3), 315-323. doi:10.1176/appi.ajp.2012.12060840.

White, S. F., Fowler, K. A., Sinclair, S., Schechter, J. C., Majestic, C. M., Pine, D. S., et al. (2014). Disrupted expected value signaling in youth with disruptive behavior disorders to environmental reinforcers. Journal of the American Academy of Child and Adolescent Psychiatry, 53(5), 579-588. doi:10.1016/j.jaac.2013. 12.023. e579.

Willcutt, E. G., Doyle, A. E., Nigg, J. T., Faraone, S. V., \& Pennington, B. F. (2005). Validity of the executive function theory of attentiondeficit/hyperactivity disorder: a meta-analytic review. Biological Psychiatry, 57(11), 1336-1346. doi:10.1016/j.biopsych.2005.02. 006.

Willoughby, M., Kupersmidt, J., Voegler-Lee, M., \& Bryant, D. (2011). Contributions of hot and cool self-regulation to preschool disruptive behavior and academic achievement. Developmental Neuropsychology, 36(2), 162-180. doi:10.1080/87565641.2010. 549980.

Yang, Y., \& Raine, A. (2009). Prefrontal structural and functional brain imaging findings in antisocial, violent, and psychopathic individuals: a meta-analysis. Psychiatry Research, 174(2), 81-88. doi:10. 1016/j.pscychresns.2009.03.012.

Young, K. D., Zotev, V., Phillips, R., Misaki, M., Yuan, H., Drevets, W. C., et al. (2014). Real-time FMRI neurofeedback training of amygdala activity in patients with major depressive disorder. PLoS One, 9(2), e88785. doi:10.1371/journal.pone.0088785. 
Zelazo, P. D., \& Carlson, S. M. (2012). Hot and cool executive function in childhood and adolescence: development and plasticity. Child Development Perspectives, 6(4), 354-360. doi:10.1111/j.17508606.2012.00246.x.

Zhang, S., \& Li, C. S. (2012). Functional connectivity mapping of the human precuneus by resting state fMRI. NeuroImage, 59(4), 35483562. doi:10.1016/j.neuroimage.2011.11.023.

Zhang, J., Gao, J., Shi, H., Huang, B., Wang, X., Situ, W., et al. (2014a). Sex differences of uncinate fasciculus structural connectivity in individuals with conduct disorder. BioMed Research International, 2014, 673165. doi:10.1155/2014/673165.
Zhang, J., Zhu, X., Wang, X., Gao, J., Shi, H., Huang, B., et al. (2014b). Increased structural connectivity in corpus callosum in adolescent males with conduct disorder. Journal of the American Academy of Child and Adolescent Psychiatry, 53(4), 466-475. doi:10.1016/j. jaac.2013.12.015. e461.

Zhu, Y., Ying, K., Wang, J., Su, L., Chen, J., Lin, F., et al. (2014). Differences in functional activity between boys with pure oppositional defiant disorder and controls during a response inhibition task: a preliminary study. Brain Imaging and Behavior, 8(4), 588-597. doi:10.1007/s11682-013-9275-7. 